\title{
DISENTANGLING SIGNATURES OF SELECTION BEFORE AND AFTER EUROPEAN COLONIZATION IN LATIN AMERICANS
}

Javier Mendoza-Revilla ${ }^{1,2, \$^{*}}$, Juan Camilo Chacón-Duque ${ }^{3}$, Macarena Fuentes-Guajardo ${ }^{4}$, Louise Ormond $^{1}, \mathrm{Ke} \mathrm{Wang}^{5}$, Malena Hurtado ${ }^{2}$, Valeria Villegas ${ }^{2}$, Vanessa Granja ${ }^{2}$, Victor Acuña-Alonzo ${ }^{6}$, Claudia Jaramillo ${ }^{7}$, William Arias ${ }^{7}$, Rodrigo Barquera Lozano ${ }^{5,6}$, Jorge Gómez-Valdés ${ }^{6}$, Hugo Villamil-Ramírez $^{8,9}$, Caio C. Silva de Cerqueira ${ }^{10}$, Keyla M. Badillo Rivera ${ }^{11}$, Maria A. NievesColón ${ }^{12}$, Christopher R. Gignoux ${ }^{13}$, Genevieve L. Wojcik ${ }^{14}$, Andrés Moreno-Estrada ${ }^{15}$, Tábita Hunemeier ${ }^{10}$, Virginia Ramallo ${ }^{10,16}$, Lavinia Schuler-Faccini ${ }^{10}$, Rolando Gonzalez-José ${ }^{16}$, MariaCátira Bortolini $^{10}$, Samuel Canizales-Quinteros ${ }^{8,9}$, Carla Gallo $^{2}$, Giovanni Poletti ${ }^{2}$, Gabriel Bedoya $^{7}$, Francisco Rothhammer ${ }^{4,17}$, David Balding ${ }^{1,18}$, Matteo Fumagalli ${ }^{19}$, Kaustubh Adhikari ${ }^{20}$, Andrés Ruiz-Linares ${ }^{1,21,22 * \pi}$ and Garrett Hellenthal ${ }^{1 * \pi}$

${ }^{1}$ Department of Genetics, Evolution and Environment, and UCL Genetics Institute, University College London, London, UK

${ }^{2}$ Laboratorios de Investigación y Desarrollo, Facultad de Ciencias y Filosofía, Universidad Peruana Cayetano Heredia, Lima, Perú

${ }^{3}$ Centre for Palaeogenetics \& Department of Archaeology and Classical Studies, Stockholm University, Stockholm, Sweden

${ }^{4}$ Departamento de Tecnología Médica, Facultad de Ciencias de la Salud, Universidad de Tarapacá, Arica, Chile.

${ }^{5}$ Department of Archaeogenetics, Max Planck Institute for the Science of Human History, Jena, Germany

${ }^{6}$ National Institute of Anthropology and History, Mexico City, Mexico

${ }^{7}$ GENMOL (Genética Molecular), Universidad de Antioquia, Medellín, Colombia

${ }^{8}$ Unidad de Genómica de Poblaciones Aplicada a la Salud, Facultad de Química, UNAM-Instituto Nacional de Medicina Genómica, Mexico City, Mexico

${ }^{9}$ Universidad Nacional Autónoma de México e Instituto Nacional de Medicina Genómica, Mexico City, Mexico

${ }^{10}$ Departamento de Genética, Universidade Federal do Rio Grande do Sul, Porto Alegre, Brazil

${ }^{11}$ Department of Genetics, Stanford School of Medicine, Stanford, California, United States

${ }^{12}$ Department of Anthropology, University of Minnesota Twin Cities, Minneapolis, Minnesota, United States

${ }^{13}$ University of Colorado Anschutz Medical Campus, Aurora, Colorado, United States

${ }^{14}$ Bloomberg School of Public Health, John Hopkins University, Baltimore, Maryland, United States

${ }^{15}$ Laboratorio Nacional de Genómica para la Biodiversidad (UGA-LANGEBIO), CINVESTAV, Irapuato, Guanajuato, Mexico

${ }^{16}$ Instituto Patagónico de Ciencias Sociales y Humanas-Centro Nacional Patagónico, CONICET, Puerto Madryn, Argentina

${ }^{17}$ Programa de Genetica Humana, ICBM, Facultad de Medicina, Universidad de Chile, Santiago, Chile

${ }^{18}$ Schools of BioSciences and Mathematics \& Statistics, University of Melbourne, Melbourne, Australia

${ }^{19}$ Department of Life Sciences, Silwood Park campus, Imperial College London, Ascot, UK

${ }^{20}$ School of Mathematics and Statistics, Faculty of Science, Technology, Engineering and Mathematics, The Open University, Milton Keynes, UK

${ }^{21}$ Ministry of Education Key Laboratory of Contemporary Anthropology and Collaborative Innovation Center of Genetics and Development, Fudan University, Shanghai, China

${ }^{22}$ Aix-Marseille Université, CNRS, EFS, ADES, Marseille, France

$\S$ Current address: Human Evolutionary Genetics Unit, Institut Pasteur, UMR2000, CNRS, Paris, France. 
53 These authors jointly supervised this work

54 *Correspondence to: javier.mendoza-revilla@pasteur.fr(J.M.R); andresruiz@fudan.edu.cn

55 (A.R.L.); g.hellenthal@ucl.ac.uk (G.H.) 
Abstract

Throughout human evolutionary history, large-scale migrations have led to intermixing (i.e., admixture) between previously separated human groups. While classical and recent work have shown that studying admixture can yield novel historical insights, the extent to which this process contributed to adaptation remains underexplored. Here, we introduce a novel statistical model, specific to admixed populations, that identifies loci under selection while determining whether the selection likely occurred post-admixture or prior to admixture in one of the ancestral source populations. Through extensive simulations we show that this method is able to detect selection, even in recently formed admixed populations, and to accurately differentiate between selection occurring in the ancestral or admixed population. We apply this method to genome-wide SNP data of 4,000 individuals in five admixed Latin American cohorts from Brazil, Chile, Colombia, Mexico and Peru. Our approach replicates previous reports of selection in the HLA region that are consistent with selection post-admixture. We also report novel signals of selection in genomic regions spanning 47 genes, reinforcing many of these signals with an alternative, commonly-used local-ancestry-inference approach. These signals include several genes involved in immunity, which may reflect responses to endemic pathogens of the Americas and to the challenge of infectious disease brought by European contact. In addition, some of the strongest signals inferred to be under selection in the Native American ancestral groups of modern Latin Americans overlap with genes implicated in energy metabolism phenotypes, plausibly reflecting adaptations to novel dietary sources available in the Americas. 


\section{Introduction}

Admixed populations offer a unique opportunity to detect recent selection. In the human lineage, genomic studies have demonstrated the pervasiveness of admixture events in the history of the vast majority of human populations (Patterson et al. 2012; Hellenthal et al. 2014; Lazaridis et al. 2014). By inferring the ancestral origins of particular genetic loci in the genomes of recently admixed individuals, recent studies have provided evidence that such admixture has facilitated the spread of adaptative genetic mutations in humans. Notable examples include the transfer of a protective allele in the Duffy blood group gene likely providing resistance to Plasmodium vivax malaria in Malagasy and Cape Verdeans from sub-Saharan Africans (Hodgson et al. 2014; Pierron et al. 2018; Hamid et al. 2021), and the transmission of the lactase persistence allele in the Fula pastoralists from Western Eurasians (Vicente et al. 2019).

An ideal setting in which to test whether and how admixture contributed to genetic adaptation is Latin America. The genetic make-up of present day Latin Americans stems mainly from three ancestral populations: indigenous Native Americans, Europeans (mainly from the Iberian Peninsula), and Sub-Saharan Africans (Wang et al. 2007; Moreno-Estrada et al. 2013; MorenoEstrada et al. 2014; Homburger et al. 2015; Chacon-Duque et al. 2018; Luisi et al. 2020) that were brought together starting $~ 500$ years ago. The admixed genomes of Latin Americans are thus the result of an intermixing process between human populations that had been evolving independently for tens-of-thousands of years and that were suddenly brought together in a new environment. In this new environment, the ancestral genomes were quickly subjected to novel pressures that were largely unfamiliar from where they firstly evolved. Therefore, the genomes of Latin Americans potentially harbor signals of both older adaptations present in each of the ancestral populations, and more recent adaptations attributable to beneficial variants, e.g. introduced from a particular ancestral population, increasing rapidly in frequency post-admixture. Motivated by this, several studies have explored the genomes of admixed Latin Americans for signatures of selection, for example focusing on events occurring since the admixture event (Tang et al. 2007; Basu et al. 2008; Ettinger et al. 2009; Guan 2014; Rishishwar et al. 2015; Deng et al. 2016; Zhou et al. 2016; Norris et al. 2020; Vicuna et al. 2020). These studies have relied on an approach similar to that of admixture mapping, where the ancestry of a genomic region in each admixed individual is assigned to a particular ancestral population, a technique known as local-ancestry-inference (LAI). Loci with significantly more inferred ancestry inherited from one ancestral population are assumed to have evolved under some form of selection (Tang et al. 2007). 
118 In addition, the genetic make-up of Latin Americans offers the opportunity to detect selection in 119 their ancestral populations, as large cohorts of Latin Americans can be leveraged to reconstruct 120 genetic variation patterns in each source population. This is of particular use for exploring selection 121 in Native Americans, since Native groups are currently underrepresented in genomic studies 122 (Sirugo et al. 2019) and as a consequence only a few studies have centered on detecting adaptive 123 signals of indigenous groups from the Americas. Such studies have identified strong selective 124 signals at different genes, particularly at those related to immunity, highlighting the selective 125 pressures that Native Americans were subjected to after they entered the continent (Lindo et al. 126 2018; Reynolds et al. 2019; Avila-Arcos et al. 2020).

128 With some exceptions (Cheng et al. 2021), these studies either limited their analyses to Latin 129 Americans with high Native American ancestry or used LAI to infer loci in individuals that derive 130 from a Native American source. However, such approaches may result in a reduction of statistical 131 power due to removal of individuals with non-Native ancestry, inaccurate local ancestry estimation 132 and/or through removing segments challenging to assign.

134 Here we present a novel statistical model that identifies loci that have undergone selection before or after an admixture event (which we refer to as pre- or post-admixture selection, respectively). In contrast to previous methods, this approach is based on allele frequencies and does not require assignments of local ancestry along the genome. We illustrate the utility of our new method by performing a selection scan in five Latin American cohorts collected as part from the CANDELA

139 Consortium (Ruiz-Linares et al. 2014). Our results suggest that several loci have been subjected to 140 natural selection in admixed Latin American populations, and in their ancestral populations, 141 replicating many of these signals using LAI. Many of the putative selected SNPs are strongly associated to relevant phenotypes, or act as expression quantitative loci (eQTL) in relevant tissues,

143 providing further evidence of their functional effect. Overall, our analyses highlight the usefulness 144 of our method to detect signals of selection in admixed populations or their ancestral populations, 145 and reveal novel candidate genes implicated in the adaptive history of groups from the American 146 continent.

\section{Results}

\section{Overview of AdaptMix}

151 In part following Balding and Nichols (1995), and analogous to previous approaches (Long 1991; 152 Mathieson et al. 2015; Cheng et al. 2021), our model AdaptMix assumes that, under neutrality, the 
153 allele frequencies of an admixed target population can be described using a beta-binomial model, 154 with expected allele frequency equal to a mixture of sampled allele frequencies from a set of groups 155 that act as surrogates to the admixing sources (fig. 1). In our case the admixed target population is a 156 Latin American cohort, defined below, and we use three surrogate groups to represent Native 157 American, European, and African admixing source populations. The mixture values are inferred a 158 priori, e.g. using ADMIXTURE (Alexander et al. 2009) (fig. 1a) or SOURCEFIND (Chacon-Duque 159 et al. 2018), as the average amount of ancestry that each admixed target individual matches to a set 160 of reference populations. (The reference populations used by these programs may be the same as the 161 surrogate populations, but they need not be as illustrated below.) We find the variance parameter 162 that maximises the likelihood of this beta-binomial model across all SNPs. This variance term aims 163 to limit the number of false-positives attributable to genetic drift in the target population following 164 admixture and/or the use of inaccurate surrogates for the ancestral populations. Then, at each SNP, 165 we calculate the probability of observing allele counts equal to or more extreme than those observed 166 in the target population, hence providing a $P$-value testing the null hypothesis that the SNP is 167 neutral (see Methods).

Assuming a pulse of admixture, this test is designed to detect selection occurring: (i) in the admixed population following the admixture event (e.g. along the purple line time period in fig. 1b), and/or

171 (ii) in one (or more) of the source/surrogate pairings, i.e. following the split of the surrogate 172 population from the admixing source it is representing (e.g. along the red and/or blue lines in fig. 173 1b). At SNPs with evidence of selection (i.e. low $P$-values), we distinguish between (i) and (ii) by 174 exploring how genotype counts of admixed target individuals relate to their inferred admixture proportions contributed by each surrogate. Under scenario (i), we assume that selection affects all target individuals equally, regardless of their admixture proportions, which in turn assumes all ancestries were present when selection occurred. In contrast, under scenario (ii), we expect selection to more strongly affect one of the source/surrogate population pairings. Intuitively, if (ii) is true, individuals with nearly $100 \%$ ancestry from the source/surrogate pair experiencing selection will 180 have genotype counts that deviate the most from expectations under the neutral model, while 181 individuals with nearly $0 \%$ ancestry from this pair will have counts that closely follow the neutral 182 model (fig. 1c). If instead (i) is true, this pattern is attenuated, though it can be challenging in 183 practice to distinguish (ii) from (i) if allele frequencies strongly differ between surrogate groups (fig 184 1d). Assuming a multiplicative model of selection, we find the selection coefficients that maximize the fit of the data to model (i) and to model (ii) when separately treating each source/surrogate pair 186 as the selected group. We report ratios of likelihoods, equivalent here to using differences in Akaike Information Criterion (AIC), to quantify our ability to distinguish among scenarios (i) and (ii). 
In summary, for each tested SNP we infer (a) a $P$-value testing the null hypothesis of neutrality, (b) the relative evidence (i.e. likelihood ratios) for whether selection occurred post-admixture or in one of the admixing sources and (c) the selection strength summed across time.

\section{Simulations}

194 We tested our approach using simulations designed to resemble our Latin American cohort in terms of sample size, inferred admixture proportions, and the extent to which our surrogates match the true admixing sources (see Methods). At a false-positive rate of $5 \times 10^{-5}$, these simulations indicate we have $\sim 50-90 \%$ power to detect selection for scenario (i) (i.e., post-admixture selection) with selection strength $(s)$ of 1.15-1.20 per generation in homozygotes carrying two copies of the selected allele, and selection occurring over 12 generations under various modes of selection

200 (additive, dominant, multiplicative, recessive) (fig. 2a, supplementary fig. S1). For scenario (ii), in 201 the case of selection occurring in the Native American source, power depends on the overall amount 202 of Native American ancestry (fig. 2a). As an example, Brazil-like simulations $(<15 \%$ average 203 Native American ancestry) show little power, Colombia-like simulations ( $30 \%$ average Native American ancestry) typically exhibit $>50 \%$ power, and other simulated populations ( $\sim 50-70 \%$ average Native American ancestry) exhibit $>75 \%$ power under scenario (ii) assuming $s=1.1$ per generation over 50 generations, with similar power if instead $\mathrm{s} \sim 1.025$ over 150 generations (supplementary fig S2). Detecting selection occurring in the European source depends on the overall amount of European ancestry in a similar manner (e.g., fig. 2a, supplementary fig. S3). For SNPs where we detect selection, we mis-classify the type of selection $\leq 2 \%$ of the time, e.g., concluding post-admixture selection when the truth is selection in the Native American source $\sim 1 \%$

211 of the time across all selection coefficients (fig. 2b). However, our approach often fails to classify selection scenarios unless selection strengths are large (e.g., $s>1.1$ ).

\section{Applying AdaptMix to the five Latin American cohorts of CANDELA}

215 We divided Latin Americans into five cohorts based on country of origin: Brazil ( $\mathrm{n}=190)$, Chile $216(\mathrm{n}=896)$, Colombia $(\mathrm{n}=1125)$ Mexico $(\mathrm{n}=773)$, and Peru $(\mathrm{n}=834)$, using individuals sampled as part 217 of the CANDELA Consortium (Ruiz-Linares et al. 2014), testing each cohort for selection 218 separately (supplementary fig. S4). Analyzing each cohort by country of origin results in a higher 219 number of individuals, and thus increases the statistical power to detect selection. As demonstrated in Chacon-Duque et al (2018), however, there is notable population sub-structure within each country. To test for robustness of our selection signals to this sub-structure, we supplemented each of these analyses by testing subsets of individuals within a country based on their inferred ancestry 
matching to Native American reference groups from Chacon-Duque et al. (2018). This gave six additional tested groups with sufficient ancestry represented: 'Mapuche' $(n=434)$ in Chile, 'Chibcha Paez' $(n=200)$ in Colombia, 'Nahua' $(n=466)$ and 'South Mexico' $(n=78)$ in Mexico, and 'Andes Piedmont' ( $\mathrm{n}=195)$ and 'Quechua' ( $\mathrm{n}=147)$ in Peru (supplementary fig. S5). To infer the proportion of African, European, and Native American ancestry in each Latin American, we applied unsupervised ADMIXTURE with $K=3$ clusters jointly to all CANDELA individuals and 553 Native American, Iberian, and West African reference individuals (fig. 1a).

230 Note that the choice of surrogate populations defines the selection test between each surrogate and 231 its corresponding ancestral source in scenario (ii). In this way, our test acts as an analogue to FST comparing two populations, but while accounting for admixture in one of the populations. As an illustration, we tested the Brazilian cohort for selection using northwest Europeans from England and Scotland (GBR) from the 1000 Genomes Project (1KGP) (The 1000 Genomes Project Consortium 2015) as a surrogate for the Brazilian cohort's European ancestry source (supplementary fig. S6). Given the majority ( $80 \%)$ of ancestry in the Brazilian cohort is related to Iberian Europeans, this test is most-powered to detect selection acting along the branch separating present-day northwest Europeans and descendants of Iberians who traveled to Brazil postColumbus. In this analysis, we infer strongest signals of selection at the HERC2/OCA2 and LCT/MCM6 genes. This replicates previously reported selection signals when comparing northwest Europeans to present-day Iberians (Poulter et al. 2003; Bersaglieri et al. 2004), and likely indicates selection for lighter skin pigmentation and lactase persistence in northwest Europeans that is unrelated to any selection in the Americas. As another example, we also tested each Latin American cohort separately while using Han Chinese from Beijing (CHB) from the 1KGP as a surrogate for Native American ancestry (supplementary fig. S7). In this analysis, SNPs that follow model (ii) indicate selection along the branch separating present-day Han Chinese and Native American populations. For this test, we find the strongest signals of selection at previously reported selected genes in East Asians, including those related to alcohol metabolism such as $A D H 7$ and $A D H 1 B$ (Galinsky et al. 2016; Gu et al. 2018) that both are classified as selection under model (ii). The strongest overall signal in this analysis overlapped the $P O U 2 F 3$ gene, implicated in the regulation of viral transcription, keratinocyte differentiation and other cellular events, which has been reported to be under selection in Native American populations from throughout the Americas (Amorim et al. 2017).

For our main analyses, we use 205 Iberians (from 1KGP and Chacon-Duque et al. (2018)) to represent European ancestry surrogates. Therefore, given the likely short split time between presentday Iberians and Europeans that migrated to the Americas during the colonial era, we are underpowered to detect selection in the European source only (see simulations). We use 206 West 
Africans from the 1KGP to represent the African ancestry source, which has been reported as a good proxy to the African genetic sources (from Chacon-Duque et al. (2018)). For this reason, we should similarly have low power to find selection occurring only in the African source/surrogate. At any rate we do not test for selection related to African ancestry, because the Latin American cohort here have $\sim 6 \%$ African ancestry on average, limiting power further. We combined 142 individuals with $<1 \%$ non-Native American inferred ancestry from 19 Native American groups (supplementary table S1) to represent the Native American surrogate. By using individuals sampled from geographically spread Native American groups as the Native American ancestry surrogate, we aim to identify regional selection signals experienced by some Native American groups but not others. We also expect to have the highest power when testing for selection type (ii) in Native Americans, as there is likely to be the most time separating this 'average' Native American surrogate and the admixing source of each regional Latin American cohort. To avoid confounding our inference, we excluded individuals with $>1 \%$ inferred ancestry matching to surrogates other than Native Americans, Iberian Europeans, and West Africans using SOURCEFIND (Chacon-Duque et al. 2018). Also, since the time since admixture among these groups is relatively short in the CANDELA cohort (likely $<15$ generations ago), detecting selection post-admixture can only identify relatively strong selection signals (see simulations).

\section{AdaptMix identifies 47 regions of putative selection}

277 For each Latin American cohort, we considered SNPs under selection as those having $P$-values less than the $5 \times 10^{-5}$ false-positive threshold in the population-matched neutral simulations, which

280 Colombia, Mexico and Peru, we report loci that pass these criteria both in the analysis of all individuals from that country and in at least one of three alternative analyses for that country that are designed to test for robustness to latent population structure (supplementary fig. S8). The first of these alternative analyses consisted of identifying signals of selection using AdaptMix on each of the six Native American subsets defined above (e.g., in either the 'Andes Piedmont' or 'Quechua' subset when testing for selection in Peruvians) (supplementary table S3). The other two alternative analyses were based on LAI. In particular we used ELAI (Guan 2014) to assign each genomic region of an admixed individual to a Native American, European, or African ancestral source. For the second alternative analysis, designed to test for post-admixture selection, we assessed whether the proportion of ancestry inferred from one of these three sources in a local region deviated substantially from the genome-wide average (supplementary table S4). For the third alternative analysis, designed to test for selection in the Native American source, we instead used the Population Branch Statistic (PBS) (Yi et al. 2010) to test for selection in one of the six Native 
American subset groups defined above, using allele frequencies computed from LAI-inferred

294 Native American segments from the subset of individuals representing that Native American group (see Methods) (supplementary fig. S5 and supplementary table S5).

Overall, we find 51 candidate regions to have evidence of positive or purifying selection passing the criteria above, 47 of which target protein-coding genes (supplementary table S6 and fig. 3). Four of these 47 candidate gene regions contain at least one SNP exhibiting strong evidence (likelihood ratio $>1,000$ ) of selection affecting all admixed individuals regardless of ancestry proportions, which we assume reflects post-admixture selection. Furthermore, 18 of these 47 regions exhibit strong evidence of selection containing at least one SNP (likelihood ratio $>1,000$ ) in the Native American source only. The 25 remaining candidate gene regions are unclassified into either type of selection (likelihood ratio $\leq 1,000$ ).

To prioritize candidate casual genes, we annotated the protein-coding gene that had the highest overall Variant-to-Gene (V2G) scores (Ghoussaini et al. 2021) for the SNPs showing the strongest evidence of selection in each candidate gene region. The overall V2G score aggregates differentially weighted evidence of variant-gene association from several sources, including cisQTL data, chromatin interaction experiments, in silico function predictions (e.g., Variant Effect

311 Predictor from Ensembl), and distance between the variant and each gene's canonical transcription 312 starting site. For each of these candidate genes we then annotated the phenotype with the highest 313 overall association score based on the Open Targets Platform (Koscielny et al. 2017).

315 While most of these associated phenotypes represent genetic disorders, syndromes, or different types of measurements (medically or non-medically-related), many are also related to immune response and diet - two major selective forces that shape the human genome (Karlsson et al. 2014;

318 Fan et al. 2016). We therefore organize the description of our candidate selection signals into two 319 main sections below that cover only these two features, with signals of all other hits in 320 supplementary table S6. For brevity, below we only highlight putatively selected regions where at 321 least one significant SNP had an associated GWAS or eQTL signal. For our significant SNPs 322 related to immune-response genes, GWAS signals included SNPs associated to white blood cell counts in a large multi-continental cohort (including Latin American individuals) (Chen et al. 324 2020), and eQTL signals included cis-associated SNPs to gene expression in 15 immune-related cell 325 types from the DICE project (Schmiedel et al. 2018). For our significant SNPs related to diet, 326 GWAS signals included metabolic, anthropometric, and lipid levels from the UK Biobank cohort 
(Loh et al. 2018), and eQTL signals included cis-associated SNPs to gene expression in adipose, muscle, and liver tissue from the GTEx Project (Lonsdale et al. 2013).

\section{Signals at immune-related genes}

331 Fifteen of the 47 candidate gene regions contained at least one protein-coding gene either related to 332 the development or regulation of the immune system or that has been previously associated to the 333 quantification of immune cell types, susceptibility progression to infectious diseases, or 334 autoimmune disorders. For example, we replicate a well-known signal encompassing several 335 immune-related genes at 6p21 that are part of the human leukocyte antigen (HLA) system (fig. 4 and supplementary fig. S9-S11). These included SNPs (AdaptMix $P$-value $<5.00 \times 10^{-7}$ ) near several MHC class I genes ( $H L A-G, H L A-H, H L A-A$, and $H L A-J)$ in each of the Chilean, Colombian, Mexican and Peruvian cohorts, with the Colombian cohort containing several SNPs classified as being selected post-admixture (likelihood ratio>1,000). Encouragingly, we inferred African ancestry enrichment (Z-score $>2.5$ ) in each cohort $\sim 60 \mathrm{~kb}$ downstream from our top AdaptMix signals using LAI, with maximum Z-score $>9$ (one-sided $P$-value $<4.09 \times 10^{-21}$ ) in the Chilean cohort (fig. 4). In addition, other signals were inferred upstream in the Chilean cohort at a 5' UTR SNP of the $Z B T B 12$ gene (rs2844455, AdaptMix $P$-value $=5.45 \times 10^{-8}$ ), the Mexican cohort at an intronic SNP of $H L A-D M A$ (rs28724903, AdaptMix $P$-value $\left.=3.87 \times 10^{-8}\right)$, and the Peruvian cohort at an intronic SNP of the MHC class III gene STK19 (rs6941112, AdaptMix $P$-value $=7.57 \times 10^{-9}$ ). Many of these HLA genes have been previously characterized as subject to be under selection postadmixture in different Latin American populations by showing an excess of African ancestry at the HLA locus (Tang et al. 2007; Basu et al. 2008; Ettinger et al. 2009; Guan 2014; Rishishwar et al. 2015; Deng et al. 2016; Zhou et al. 2016; Norris et al. 2020; Vicuna et al. 2020).

In addition to HLA, we infer previously unreported selection signals in four candidate gene regions that each harbor genes with well-established roles in the immune system, with each region containing at least one SNP significantly associated $\left(P\right.$-value $\left.<5 \times 10^{-8}\right)$ to white blood cell counts or the expression of an immune-related gene in immune cells $\left(P\right.$-value $\left.<10^{-5}\right)$ (see Methods). Among these, one signal at $1 \mathrm{p} 13$ in the Chilean cohort encompasses the CD101 gene (fig. 5a), which belongs to a family of cell-surface immunoglobulins superfamily proteins and plays a role as an inhibitor of T-cell proliferation (Soares et al. 1998; Bouloc et al. 2000). Within this region five SNPs are classified as being selected post-admixture and show also an increase of LAI-inferred European ancestry (maximum $Z$-score $=3.40$, one-sided $P$-value $=3.36 \times 10^{-4}$ ). Strikingly, the region contains a synonymous SNP (Ile588, CADD score of 9.23) (rs3736907, AdaptMix P361 value $=1.05 \times 10^{-9}$ ) that strongly affects $C D 101$ expression in T cells (eQTL $P$-value $<2.42 \times 10^{-10}$ ) 
and is associated with neutrophil (GWAS $P$-value $=2.08 \times 10^{-10}$ ) and total white cell count (GWAS $P$-value $\left.=3.61 \times 10^{-9}\right)$ (fig. 5a).

The second signal, at 18p11 also in Chileans, encompasses the PTPN2 gene, a tyrosine-specific phosphatase involved in the Janus kinase (JAK)-signal transducer and activator of transcription (STAT) signaling pathway (fig. 5b). The JAK-STAT pathway has an important role in the control of immune responses, and dysregulation of this pathway is associated with various immune disorders (Shuai and Liu 2003). Several SNPs with low AdaptMix $P$-values $\left(P\right.$-value $\left.<1.69 \times 10^{-7}\right)$ in the 18 p11 region are also associated with eosinophil counts (GWAS $P$-value $<1.13 \times 10^{-10}$ ) and the expression of PTPN2 in natural killer (NK) cells (eQTL $P$-value $\left.<1.14 \times 10^{-9}\right)$ (fig. $5 \mathrm{~b}$ ).

The other two novel signals, both in the Peruvian cohort, are consistent with selection in Native Americans only (likelihood-ratio $>1,000$ ). The first, at 17q25, contains the CD300LF gene that encodes for a membrane glycoprotein that contains an immunoglobulin domain, and which plays an important role in the maintenance of immune homeostasis by promoting macrophage-mediated efferocytosis (Borrego 2013). Notably, a 3'UTR SNP (rs9913698, AdaptMix $P$-value $=3.11 \times 10^{-9}$ ) is strongly associated with monocyte count (GWAS $P$-value $=1.00 \times 10^{-33}$ ), total white cell count (GWAS $P$-value $=5.96 \times 10^{-24}$ ), lymphocyte count (GWAS $P$-value $=2.50 \times 10^{-19}$ ), and neutrophil count (GWAS $P$-value $\left.=1.30 \times 10^{-9}\right)$ (supplementary fig. S12). The second signal is at $22 \mathrm{q} 11$ adjacent to the MIF gene (fig. 5c), which is implicated in macrophage function in host defense through the suppression of anti-inflammatory effects of glucocorticoids (Calandra and Roger 2003). Variants within MIF have been recently associated to rheumatoid arthritis in southern Mexican patients

384 (Santoscoy-Ascencio et al. 2020). The SNP rs2330635 (AdaptMix $P$-value $=7.06 \times 10^{-8}$ ) is strongly associated to the expression of MIF in T-cells (eQTL $P$-value $<8.63 \times 10^{-5}$ ) and NK cells (eQTL $P$ value $\left.=5.77 \times 10^{-9}\right)$ and is also marginally associated to neutrophil counts (GWAS $P$-value $=2.46 \times 10^{-}$ $\left.{ }^{6}\right)$ (fig. 5c).

Overall, these findings suggest that some of the most robust signals of adaptation in the Americas can be ascribed to immune-related selective pressures. These plausibly resulted from both the introduction of novel pathogens after European colonization and the endemic pathogens encountered by the first Native Americans during the initial peopling of the continent.

\section{Signals at genes related to diet}

Among the 47 candidate regions, nine regions contained at least one protein-coding gene potentially related to dietary practices through their association with metabolism-related phenotypes or 
anthropometric-related measurements (supplementary table S6). Among these, we infer three previously unreported signals where at least one of the selected SNPs was associated to metabolicor anthropometric-related phenotypes, or to the expression of the candidate gene in adipose, muscle, or liver tissue (see Methods). One of these three hits (rs4636058, AdaptMix $P$-value $=5.70 \times 10^{-10}$ ), at $6 \mathrm{p} 22$ in the Chilean cohort, is classified as being selected post-admixture and shows an increase of LAI-inferred European ancestry $\left(Z\right.$-score $=3.78$, one-sided $P$-value $\left.=7.82 \times 10^{-4}\right)$. It is located at $6 \mathrm{q} 22$ and encompasses the $S L C 35 F 1$ gene, whose function is not known, though several studies have associated this gene with different measurements of cardiac function (Hoffmann et al. 2017; Warren et al. 2017; Giri et al. 2019). Notably, SNP rs4636058 is marginally associated to cholesterol levels (UKBB GWAS $P$-value $=3.8 \times 10^{-4}$ ) and body fat percentage $\left(\right.$ UKBB GWAS $P$-value $=4.29 \times 10^{-4}$ ). Another of these three hits, at 1q31 in the Mexican cohort, is consistent with selection in Native Americans (likelihood-ratio>1,000) (fig. 6a). The 1q31 signal includes an intronic SNP (rs1171148, AdaptMix $P$-value $=2.31 \times 10^{-8}$ ) of $B R I N P 3$, a gene associated to body mass index in studies across different human groups (Pulit et al. 2019; Zhu et al. 2020). Within this region, various SNPs are associated to different metabolic-related phenotypes, including the SNP rs1171148 that is associated with hip circumference (UKBB GWAS $P$-value $=4.96 \times 10^{-8}$ ) and marginally associated with body mass index (UKBB GWAS $P$-value $\left.=5.51 \times 10^{-5}\right)$ (fig. 6 a).

Finally, the third hit (rs5030938, AdaptMix $P$-value $\left.=3.79 \times 10^{-15}\right)$, which had the highest overall AdaptMix score, is inferred in the Peruvian cohort at 10q22 and indicates selection in Native Americans (likelihood-ratio $>1,000$ ) (fig. 6b). This SNP is associated with the expression of $H K D C 1$ in liver (eQTL $P$-value $=2.19 \times 10^{-5}$ ), adipose visceral (eQTL $P$-value $=1.46 \times 10^{-5}$ ), and adipose subcutaneous tissue (eQTL $P$-value $=1.36 \times 10^{-4}$ ) (fig. 6b). $H K D C 1$ encodes and hexokinase that catalyzes the rate-limiting and first obligatory step of glucose metabolism (Ludvik et al. 2016), and several studies have associated variants within this gene with glucose levels in pregnant women (Hayes et al. 2013; Guo et al. 2015; Kanthimathi et al. 2016; Tan et al. 2019) and with weight at birth (Warrington et al. 2019).

Overall, these results support previous hypothesis that genes related to energy metabolism were probably critical in the establishment of stable human populations in distinct ecoregions (Hancock

\section{Discussion}


432 Here we present AdaptMix, a novel statistical model that identifies loci under selection in admixed 433 populations. Our model is based on the principle that allele frequencies in an admixed population 434 can be modeled as a linear combination of the allele frequencies in the ancestral populations 435 proportional to their admixing contributions, and that deviations from the expectation can be a 436 product of selection. This selection test is related to the work of Long (1991) and Mathieson et al. 437 (2015). One difference is that our approach directly infers and models the variance of the predicted 438 allele frequencies in the admixed population given the set of surrogates used for ancestral sources. 439 This parameter can help control for large deviations in allele frequency arising solely from genetic 440 drift experienced in the admixed population (Long 1991; Bhatia et al. 2014) and/or from using 441 inaccurate proxies for one or more of the source populations. In some applications here, e.g. the 442 Brazilian cohort, AdaptMix gives $P$-values with a median near 0.5 as expected under the null 443 hypothesis of neutrality, indicating a correction approach such as genomic control may not be 444 necessary as in Mathieson et al. (2015) (supplementary fig. S13). However, simulations under 445 neutrality that follow a slightly different model than our inference approach (see Methods), shows 446 AdaptMix gives both an excess of high and low $P$-values relative to the uniform distribution 447 expected under neutrality (supplementary fig. S14). This suggests our $P$-values are not well448 calibrated, perhaps reflecting deviations from the underlying model and necessitating caution when 449 choosing thresholds for significance. We thus based our significance thresholds on neutral 450 simulations tailored to each cohort, and focus only on the strongest association signals that resulted 451 in low false-positive rates based on simulated neutral SNPs. However, we caution that necessarily 452 simulations are over-simplifications of complex latent demographic processes, and more work is 453 required to verify these signals.

455 Another important contribution of our test is that it can infer whether selection disproportionately 456 affects one source/surrogate pairing or affects all ancestry backgrounds equally. We assume 457 selection affecting all ancestry backgrounds indicates selection occurring post-admixture, which is 458 more parsimonious than an alternative explanation of independent selection events differentiating 459 allele frequencies between each admixing source and its surrogate. For inferred selection in a 460 source/surrogate pairing, this can reflect selection occurring in that source and/or its surrogate, 461 possibly even following the admixture event. Post-admixture selection affecting only one source 462 may be possible in cases of selection only occurring in a particular environment that is correlated 463 with admixture fractions. For example, selection we detect to occur in Native Americans may be 464 attributable to Europeans introducing a new environmental pressure (e.g. infectious disease) that 465 disproportionately affected fitness in indigenous Americans. However, the split time between the 466 true Native American ancestral source and our Native American surrogate is likely much longer 
than the time since colonial era admixture, suggesting selection pre-admixture as a more plausible explanation given the longer time to act. Supporting this, our inferred selection coefficients (which are summed over time) in cases where we conclude selection in Native Americans are typically greater than 2 (supplementary table S6). If selection had occurred post-admixture continuously over the last 12 generations (corresponding to an admixture date of $\sim 1650 \mathrm{CE}$ ), this value approximately corresponds to a per generation selection coefficient $\sim 0.16$, which is strong relative to previous reports of recent selection in human populations (e.g. Hamid et al. (2021)). In contrast, our four signals concluding post-admixture selection infer a per generation selection coefficient $<0.1$, which falls more in line with previous inference of selection strengths.

For 18 genomic regions where we conclude selection in the Native American source (supplementary table S6), it is possible this is capturing selection in (some subset of) groups that comprise the Native American surrogate group we use here, rather than in the (more localized) Native American source of the admixed population. The lack of overlap in selection signals when analysing the five CANDELA cohorts, and lack of concordance of our signals with those from PBS testing for selection in this combined Native American surrogate (supplementary fig. S15), suggests our signals are not being driven by selection in this combined population in practice. Furthermore, when using PBS to test for selection in LAI-inferred Native American segments from individuals with high degrees of ancestry recently related to the tested Native American source, an analysis that does not use the combined Native American surrogate, PBS scores for SNPs in 6 of these 18 regions fall into the top $99.99^{\text {th }}$ percentile (supplementary fig. S16-21), with the remaining 13 regions containing SNPs in the top $99^{\text {th }}$ percentile. However, relative to our approach, LAI-based inference (e.g., Avila-Arcos et al. (2020)) may be more robust to using combined data from multiple populations to represent one surrogate, since it only requires matching to a subset of individual's haplotype patterns in the reference panel.

In general our approach has decreased power to distinguish whether selection occurred postadmixture versus in one of the ancestral sources, if reference population allele frequencies are very different and selection is weak (fig. 1c). Inferring excess ancestry matching using LAI would likely better capture post-admixture selection in such cases, e.g. a scenario where one population that is fixed (or nearly-fixed) for the protective allele intermixes with a population nearly-fixed for the non-protective allele, with the admixed population subsequently undergoing selection. An example of this is a recently reported excess of African ancestry, likely attributable to post-admixture selection, on the Duffy-null allele in inhabitants of Santiago Island in Cape Verde (Hamid et al. 2021). However, our test to detect whether any type of selection occurred should not be affected by 
these scenarios. In addition, our approach may identify post-admixture selection in scenarios that excess-ancestry LAI-based would miss by design, such as cases where the selected allele is at a similar frequency in all reference populations. Perhaps the most important contrast to LAI and other approaches detecting selection in admixed populations (Cheng et al. 2021), is that in principle our approach can be applied to populations that descend from the mixture of genetically similar groups, e.g. if using haplotype-based approaches (e.g. SOURCEFIND) to infer ancestry proportions. Future work should assess the power of this technique under such admixture settings.

While our method assumes a single pulse of admixture, theoretically our ability to diagnose and classify selection occurring in only one source should not be affected by multiple instances of (or continuous) admixture from that or any other source. This is because the signal of allele frequency deviation due to selection in such cases is entirely determined by the amount of ancestry inherited from that source, and not the number of admixture pulses. In contrast, if an admixed population experiences selection and then receives new migrants from one of the original admixing sources that are unaffected by this selection, e.g. later European migrants to the Americas, in theory this may attenuate our ability to determine that selection occurred post-admixture. However, in a simple scenario of one such additional admixture pulse, contributing $10-50 \%$ of DNA, the correct postadmixture selection theoretical model fits as well or better to the theoretical truth than does the incorrect model concluding selection in the source that did not contribute new migrants (supplementary fig. S22).

As noted above, and consistent with other tests comparing populations (Mathieson 2020), the choice of surrogate group can make a difference in the inferred selection signals. For example, our largest signal of Native American selection, at 10q22 and most strongly signalled in the "Andes Piedmont' Peruvian subgroup, disappears if replacing the 'combined Native American' surrogate group with Han Chinese (CHB from the 1KGP) (supplementary fig. S7). In this case, the frequency of the putatively selected allele (rs5030938) is 67\% in LAI-inferred Native haplotypes in the Peruvian 'Andes Piedmont' subgroup, which is notably higher than the 38-54\% observed in LAIinferred Native American haplotypes in four non-Peruvian sub-groups, and thus consistent with selection (supplementary table S7). However, it is lower than that of $\mathrm{CHB}(\sim 76 \%$,), which explains the lack of signal when using $\mathrm{CHB}$ as a surrogate. The frequency in Yakut, a Siberian group that perhaps better represents ancestral Native Americans than CHB does (Wang et al. 2007), is closer to that of frequency estimates across non-Peruvian Native American groups (0.46-0.5). In general, there is a trade-off between using surrogates more distantly related to the source, which may decrease power to find regional adaptation signals, versus choosing a more closely related 
surrogate, which may also decrease power by masking adaptation signatures that it shares with the target source (e.g. using Iberians as a surrogate for European ancestry of Latin Americans). Our inferred variance parameter can be used to investigate how well a given surrogate captures genetic variation in the target population, with for example the inferred variance using $\mathrm{CHB}$ as a surrogate $\sim 5$-10-fold higher relative to using the combined Native American surrogate.

\section{Selection signals detected in the CANDELA cohort}

544 The candidate genes we infer to be affected by selection in Latin Americans and their Native 545 American ancestors are best viewed in the context of other previously reported signals. Reynolds et 546 al. (2019) recently performed a selection scan in three Native North American populations and identified some of the strongest signals at immune-related genes including the interleukin 1 receptor Type $1(I L 1 R l)$ gene in a sample from several closely related communities in the southeastern United States, and the mucin 19 (MUC19) gene in a central Mexican population. We do not replicate the MUC19 signal in the CANDELA Mexican cohort, which could indicate that the Native American component in this cohort is not closely related to that of the central Mexican Native American group. Nonetheless, we found some of our strongest signals of selection at several loci encompassing genes involved in the immune response, including $C D 300 L F$ and $M I F$, detected as being selected in the Native American ancestors of Peruvians. Interestingly, CD300LF promotes macrophage-mediated efferocytosis, while MIF play a role regulating macrophage function through the suppression of glucocorticoids. These observations suggest that these two genes might have perhaps evolved in a coordinated manner, possibly due to their phagocytic-related role against the novel pathogens encountered in the Americas.

Regarding signals of selection post-admixture, several studies have consistently shown adaptive signals in different Latin American populations at HLA by showing an excess of matching to African reference haplotypes using LAI (Tang et al. 2007; Basu et al. 2008; Ettinger et al. 2009; Guan 2014; Rishishwar et al. 2015; Deng et al. 2016; Zhou et al. 2016; Norris et al. 2020; Vicuna et al. 2020). Given that African ancestry was enriched at this region, the authors suggested that certain African alleles could have conferred a selective advantage to certain infectious diseases most likely brought by Europeans. While AdaptMix is only able to classify selection in one cohort (Colombia) out of our four HLA signals, we also replicated this excess of African ancestry in each of the CANDELA cohorts (supplementary fig. S9). There is some debate as to whether these signals are genuine or attributable to confounders such as inaccurate LAI inference (Pasaniuc et al. 2013). To illustrate the validity of these concerns, people with entirely Northwest European ancestry from 
genuine selection at HLA in Northwest Europeans - presumably does not reflect genuine recent African ancestry (supplementary fig. S23). Instead this is more likely attributable to the relatively high degree of genetic diversity in HLA mimicking African genetic diversity, illustrating how these LAI-based tests can give false-positive signals when testing for post-admixture selection. This may explain why AdaptMix does not replicate the moderate amount of excess African ancestry inferred by LAI at HLA in the Brazilian cohort (supplementary fig. S9), which is predominantly of European ancestry. Indeed regions under selection in admixed populations may be particularly difficult to classify accurately using LAI, e.g. with the HLA region here having the lowest overall LAI classification probability (supplementary fig. S24), especially in cases where the reference population have not experienced similar selection and hence may have poorly matching genetic variation patterns. As our approach does not require LAI, it is robust to these issues. While our model is not able to classify selection as post-admixture at most of our HLA signals, allele frequency patterns in the admixed cohorts are consistent with post-admixture selection and often show allele frequencies drifting away from those expected under our neutral model and towards those of the African or European reference population (supplementary fig. S25). This is most evident in the Colombian cohort, consistent with Africans contributing protective alleles as previously suggested (Tang et al. 2007; Basu et al. 2008; Ettinger et al. 2009; Guan 2014; Rishishwar et al. 2015; Deng et al. 2016; Zhou et al. 2016; Norris et al. 2020; Vicuna et al. 2020). In addition to HLA, we also identified a novel post-admixture selection signal in the Chilean cohort that was accompanied by a significant increase of European ancestry at the CD101 locus, again, suggesting that protective alleles from Europeans might have also been adaptive to counter Old World-borne diseases brought to the Americas.

The signals encompassing genes related to metabolic and anthropometric-related phenotypes are consistent with novel dietary practices in the Americas driving adaptation, with many signals with an effect on relevant phenotypes and/or tissues, classified as being selected in the Native American source. Previous studies have shown evidence of adaptation at genes related to metabolic-related phenotypes and attributed the adaptation to dietary pressures in Native Americans. Avila-Arcos et al. (2020) recently reported strong signals of selection in the Mexican Huichol at several genes associated to lipid metabolism, including $A P O A 5$ and $A B C G 5$. We do not replicate these signals in the CANDELA Mexican cohort, which could indicate that the Native American component in this cohort is not closely related to that of the Huichol. The signals at APOA5 and ABCG5 are in line with a previous finding of a strong selection signal at another ATP-binding cassette transporter A1 $(A B C A 1)$ gene that has been associated with low high-density lipoprotein cholesterol in Latin Americans (Villarreal-Molina et al. 2008; Acuña-Alonzo et al. 2010). As the ABCA1 protein 
607 carrying the putative selected allele shows a decrease cholesterol efflux, the authors suggest that 608 this variant could have favored intracellular cholesterol and energy storage, which in turn might 609 have beneficially influenced the ability to accommodate fluctuations in energy supply during severe 610 famines and during the regulation of reproductive function (Acuña-Alonzo et al. 2010). Lindo et al. 611 (2018) used a genomic transect of Andean highlanders from northern Peru, and found the strongest 612 signals of selection at $M G A M$, a gene related to starch digestion. The authors attributed this finding 613 to a dietary-related selective pressure perhaps brought by the transition to agriculture in this region. 614 AdaptMix shows evidence in the CANDELA Peruvian cohort within MGAM (rs7810984, 615 AdaptMix $P$-value $=1.79 \times 10^{-8}$, above $99.9^{\text {th }}$ percentile) only when using $\mathrm{CHB}$ as a surrogate for 616 Native American ancestry. This again illustrates how the choice of surrogate populations defines the 617 selection test between each surrogate and its corresponding ancestral source. It is possible that by 618 including Andean Native Americans in our Native American source population (supplementary 619 table S1) we are affecting the power to detect selection in the Andean Native American ancestors of 620 the CANDELA Peruvian cohort, analogous to how Lindo et al. (2018) no longer detect selection at 621 MGAM if using PBS to compare ancient and present-day (Aymara) Andean groups.

Studies have also reported signals of selection in Native Americans groups shared with Siberian populations, which the authors interpreted as an adaptation to polyunsaturated-rich diets prior or close to the peopling of the Americas, likely in the Arctic Beringia. These included a signal overlapping the WARS2 and TBX15 genes, previously associated to body fat distribution and adipose tissue differentiation (Racimo et al. 2017), and the fatty acid desaturase (FADS) gene cluster that modulates the manufacture of polyunsaturated fatty acids (Amorim et al. 2017; Harris et al. 2019) (but see Mathieson (2020) for an alternative explanation of the FADS signal). Again, we inferred moderate selection evidence at these regions in the CANDELA Peruvian cohort only when using CHB as surrogate for Native American ancestry (SNP rs2361028 near TBX15, AdaptMix Pvalue $=1.8 \times 10^{-7}$, above $99.5^{\text {th }}$ percentile; SNP rs 174576 within $F A D S 2$, AdaptMix $P$-value $=3.8 \times 10^{-}$ ${ }^{8}$, above $99.5^{\text {th }}$ percentile). It is thus tempting to suggest that the three novel signals of selection AdaptMix classifies as being under selection in Native Americans might be related to dietary pressures experienced prior or during the peopling of the Americas (e.g., the BRINP3 signal detected in Mexicans), or as a product for a greater reliance of domesticated crops including potatoes (3400-1,600 CE) (Rumold and Aldenderfer 2016) (e.g., the HKDCl signal detected in Peruvians). However, it is important to note that other factors may also be attributable for some of these selection signals. 
overall selection signal within $H K D C 1$ at 10q22 (fig. 6b). Mutations within STOX1 have been associated to preeclampsia (Van Dijk et al. 2005; van Dijk and Oudejans 2011), a pathology of pregnancy characterized by high blood pressure and signs of damage to other organ system that can be lethal for the mother and for the fetus (Sibai 2003). Interestingly, in the single linkage study on preeclampsia conducted in Andean Peruvian families to date, SNPs within STOX1 show marginal association $(P$-value $=0.004678)$ (supplementary fig. S26) (Badillo Rivera and Nieves Colón et al. 2021). Given that high altitude is linked to an increased incidence of preeclampsia (Zamudio 2007), it is possible that natural selection has acted on genes related to this condition. Furthermore, the fact that variants within $H K D C 1$ are associated with glucose levels in pregnant women (Hayes et al. 2013; Guo et al. 2015; Kanthimathi et al. 2016; Tan et al. 2019) and considering the relationship between abnormal glucose levels and preeclampsia (Joffe et al. 1998; Weissgerber and Mudd 2015), it is also possible that natural selection has targeted variants at $H K D C 1$ due to its role in glucose metabolism.

Lastly, other environmental factors may also be attributable for some of these selection signals, such as infectious diseases. There is growing evidence of a link between metabolic diseases and innate immunity or inflammation (Pickup and Crook 1998; Kominsky et al. 2010; Lumeng and Saltiel 2011; Robbins et al. 2014). For instance, it has been shown that cholesterol plays a key role in various infectious processes such as the entry and replication of flaviviral infection (OsunaRamos et al. 2018). Additional studies in indigenous American populations will be needed to disentangle the putative selective pressures at these loci.

\section{Conclusion}

We have presented a novel allele frequency-based method that identifies loci under selection in admixed populations, while determining whether the selection affected all ancestral sources equally, indicating selection following admixture, or in only one of the sources. The novel candidate genes under selection provide new insights into the adaptive traits necessary for the early habitation of the Americas and to respond to the challenge of infectious pathogens corresponding to European contact. Future functional investigations will allow a more detailed understanding of the consequences of selective pressures experienced in the American continent, including its effect on present-day health outcomes. 
Materials and Methods

\section{Genomic datasets}

677 The Latin American individual samples analyzed here were part of CANDELA Consortium (Ruiz678 Linares et al. 2014). The CANDELA Consortium samples (http://www.ucl.ac.uk/silva/candela) 679 have been described in detail in previous publications (Ruiz Linares et al 2014; Chacon-Duque et 680 al., 2018). These data include a total of 6,630 volunteers from five Latin American countries 681 (Brazil, Chile, Colombia, Mexico and Peru). This dataset was genotyped on the Illumina 682 HumanOmniExpress chip platform including 730,525 SNPs. We also collated reference populations 683 from regions that have contributed to the admixture in Latin America. For Native American 684 samples we used individuals previously genotyped by Chacon-Duque et al. (2018). This dataset 685 compromises 19 Native American populations from throughout the Americas with genotype data 686 (supplementary table S1). For all the analyses described, we have only retained Native American 687 individuals that showed more than 99\% Native American ancestry as estimated by ADMIXTURE 688 (see below). For European samples, we used genotype data from Portuguese and Spanish, 689 individuals previously genotyped by Chacon-Duque et al. (2018) and Spanish (IBS; Iberian 690 Population in Spain) from the 1000 Genomes Project study (The 1000 Genomes Project Consortium 691 2015). For Sub-Saharan Africans, we used genotype data from Yoruba (YRI; Yoruba in Ibadan, 692 Nigeria), and Luhya (LWK; Luhya in Webuye, Kenya) individuals from the 1KGP. The reference 693 samples from Chacon-Duque et al. (2018) are described in more detail in the Supplementary Table 6941 from the mentioned publication. For some of our analysis we also included the 103 Han Chinese 695 from Beijing (CHB) and 85 Europeans from England and Scotland (GBR) from the 1KGP as a 696 surrogate for the Native American and European source, respectively. Genotype data of the 697 individuals from the $1 \mathrm{KGP}$ was downloaded from the 1000 Genomes Project FTP site available at ftp://ftp.1000genomes.ebi.ac.uk/vol1/ftp/.

\section{Data curation}

701 We used PLINK v1.9 (Chang et al. 2015) to exclude SNPs and individuals with more than 5\% missing data or that showed evidence of genetic relatedness as in Chacon-Duque et al. (2018). Due to the admixed nature of the Latin American samples, there is an inflation in Hardy-Weinberg $P$ values, and therefore we did not exclude SNPs based on Hardy-Weinberg deviation. After applying these filters, 625,787 autosomal SNPs and 7,986 individuals were retained for further analysis. 


\section{Selecting admixed Latin American and reference individuals}

708 In order to select admixed Latin American individuals (i.e. individuals with varying degrees of 709 Native American, European and African ancestry), we conducted an unsupervised ADMIXTURE 710 analysis at $K=3$ using a set of 103,426 LD-pruned SNPs including Native Americans, Iberian 711 Europeans and West Africans. We then removed non-admixed Latin American individuals that we 712 define as having less than $10 \%$ or more than $90 \%$ Native American genome-wide ancestry. To 713 avoid confounding our selection inference due to underlying population structure, we further 714 excluded individuals with $>1 \%$ inferred ancestry matching to surrogates other than Native 715 Americans, Iberian Europeans, and West Africans using SOURCEFIND estimates obtained for the 716 same individuals in Chacon-Duque et al. (2018). After this filtering procedure, the five Latin 717 American populations consisted of 190 Brazilians (BRA), 1125 Colombians (COL), 896 Chileans 718 (CHL), 773 Mexicans (MEX) and 834 Peruvians (PER). From our Native American, European, and 719 Sub-Saharan African reference populations, we also removed individuals that contained more than $7201 \%$ of ancestry from another group based on the ADMIXTURE analysis described above. After this 721 extra filter our final reference dataset was composed of 142 Native Americans, 205 Europeans, and 722206 Sub-Saharan Africans.

\section{Change in allele frequency under Wright-Fisher with multiplicative model of selection}

Assuming a multiplicative model of selection and random mating, the frequency of the three genotypes in generation 1 at a biallelic locus with alleles $\mathrm{A}$ and a at frequencies $p$ and $1-p$, respectively, in the previous generation is:

\begin{tabular}{c|c|c}
$A A$ & $A a$ & $a a$ \\
\hline$\left(1+s_{1}\right)^{2} p^{2} / c_{1}$ & $\left(1+s_{1}\right) 2 p(1-p) / c_{1}$ & $(1-p)^{2} / c_{1}$
\end{tabular}

where $s_{1} \in[-1, \infty]$ is the selection coefficient in generation 1 and $c_{1}=\left(1+s_{1}\right)^{2} p^{2}+$

$731\left(1+s_{1}\right) 2 p(1-p)+(1-p)^{2}$. Note that each copy of the A allele changes fitness by a factor of $732\left(1+s_{1}\right)$.

More generally, the allele frequency $p_{g}$ of allele $A$ in generation $g$ is:

$$
p_{g}=\frac{(1+s) p}{1+s p}
$$

where 


$$
s=\left[\sum_{i=1}^{g} s_{i}\right]+\left[\sum_{j=1}^{g-1}\left(s_{j} \sum_{i=j+1}^{g} s_{i}\right)\right]+\sum_{i=3}^{g} \prod_{i} \approx \sum_{i=1}^{g} s_{i}
$$

739 with $s_{i}$ the selection coefficient at generation $i$ and $\Pi_{i}$ the sum of the products of all $\left(\begin{array}{c}g \\ i\end{array}\right)$ 740 combinations of $\left\{s_{1}, \ldots, s_{i}\right\}$ values. The approximation in equation (4) assumes the $s_{i}$ are small, 741 which should be a reasonable approximation based on e.g. estimated selection coefficients in 742 humans.

\section{Testing for evidence of selection at a SNP}

745 To assess the evidence of selection at a SNP, we employ a model inspired by that used in Mathieson et al. (2015) and based on the Balding-Nichols formulation (Balding and Nichols 1995). In particular for the allele count $X_{j}$ at SNP $j$ in the target population, we assume:

$$
\operatorname{Pr}\left(X_{j}=x_{j} \mid M, p_{j}, D\right)=\operatorname{Beta}-\operatorname{Binomial}\left(x_{j} ; 2 M, \frac{1-D}{D} p_{j}, \frac{1-D}{D}\left(1-p_{j}\right)\right)
$$

where $M$ is the number of target individuals. The above model implicitly assumes that the frequency of the allele in the target population follows a Beta(mean $=p_{j}$, variance $=D p_{j}(1-$ $\left.p_{j}\right)$ ). Under neutrality, we assume

$$
\left.p_{j}=\frac{1}{M} \sum_{k=1}^{K}\left(\left[\sum_{i=1}^{M} \alpha_{k}(i)\right] f_{j k}\right)\right)
$$

where $f j k$ is the sampled frequency of the allele in the surrogate population at SNP $j$ for source $k$, and $\propto_{k}(i)$ is the inferred admixture proportion from population $k$ in individual $i$. We first find $\widehat{D}$ as the value of $D$ that maximizes $\prod_{j=1}^{J}\left[\operatorname{Pr}\left(X_{j} \mid M, p_{j}, D\right)\right]$, using the optim function in $\mathrm{R}$ with the 'Nelder-Mead' algorithm. Then, fixing $D=\widehat{D}$ in equation (3), for each SNP we find the two-sided $P$-value testing the null hypothesis that the observed allele counts follow this neutral model.

The variance under (3) is small for SNPs with very high or very low $p_{j}$, so such SNPs tend to reject this null model even in cases where the observed target population allele frequency does not deviate notably from its neutral expectation $p_{j}$ in (4). Therefore, we used an alternative parameterisation where we assumed the frequency of the allele in the target population follows a Beta(mean $=$ $p_{j}$, variance $\left.=V\right)$. This was achieved by substituting $D$ in equation (3) at $\mathrm{SNP} j$ with 
$\min \left[\frac{V}{p_{j}\left(1-p_{j}\right)}, 0.99999\right]$, necessary to ensure numerical stability, and finding $\widehat{V}$. In practice this

means that SNPs with minor allele frequency $<(1.00001 \times V)$ had variance $\left(0.99999 p_{j}\left(1-p_{j}\right)\right)$ rather than $V$, though this approach gave sensible results in practice.

\section{Determining whether selection occurred pre or post-admixture}

771 Consider the scenario in supplementary fig. S27, where sampled population $\mathrm{C}$ descends from an admixture of unsampled populations $A^{*}$ and $B^{*}$, who are represented by sampled surrogate populations A and B, respectively. Our test aims to distinguish whether selection occurred postadmixture along branch (e) versus along any of branches (a)-(d). Let $f_{c}$ be the allele frequency of a sample from population C. At a neutral SNP:

$$
E\left[\mathrm{f}_{\mathrm{c}}\right]=\alpha f_{A^{*}}+(1-\alpha) f_{B^{*}}
$$

where $f_{A^{*}}$ and $f_{B^{*}}$ are true allele frequencies of $A^{*}$ and $B^{*}$ at the SNP, respectively, and $\alpha$ is the admixture proportion from $A^{*}$. Letting $f_{k}$ be the sampled allele frequency for population $k$ serving as surrogate to the true admixing population $k^{*}$, it seems reasonable to assume:

$$
E\left[\mathrm{f}_{\mathrm{c}}\right]=\propto f_{A}+(1-\propto) f_{B}
$$

Note that this also holds under selection along branch (f) in supplementary fig. S27, which we ignore here (but which can be tested by comparing allele frequencies in $A$ and $B$ ). Equation (6) $f_{B^{*}}$, respectively, at the SNP, which may not be true. We test the effect of this using simulations, described below, in which surrogates vary in how well they reflect their respective true admixing sources.

In the case of a multiplicative model of selection along branch (e) in supplementary fig. S27 at this SNP, using equation (1) we assume:

$$
E\left[\mathrm{f}_{\mathrm{c}}\right]=\frac{(1+s)\left[\propto f_{A}+(1-\propto) f_{B}\right]}{1+s\left[\propto f_{A}+(1-\propto) f_{B}\right]} \equiv E_{c}\left[f_{c}\right]
$$


Alternatively, under a nultiplicative model for selection along branches (a) and/or (c) in supplementary fig. S27, with analogous results for selection along branches (d) and/or (b), instead we assume:

798

$$
E\left[\mathrm{f}_{\mathrm{c}}\right]=\propto\left[\frac{\left(1+s_{A}\right) f_{A}}{1+s_{A} f_{A}}\right]+(1-\propto) f_{B}=f_{B}+\propto\left[\frac{\left(1+s_{A}\right) f_{A}}{1+s_{A} f_{A}}-f_{B}\right] \equiv E_{A}\left[\mathrm{f}_{\mathrm{c}}\right]
$$

799

$$
f_{T}^{P}(i \mid s)=\frac{(1+s)\left[\sum_{k=1}^{K} \alpha_{k}(i) f_{k}\right]}{1+s\left[\sum_{k=1}^{K} \alpha_{k}(i) f_{k}\right]}
$$

where $s_{A}$ is the selection strength along branches (a) and/or (c). Importantly, $E_{A}\left[f_{c}\right]$ is linear in $\propto$, while $E_{C}\left[f_{c}\right]$, is not, which we aim to exploit to distinguish between these two scenarios.

Here, assuming CANDELA population $T$ can be described as a mixture of $K$ sources, we assume the genotype $g_{i}$ of individual $i \in[1, \ldots, M]$ from $T$ follows:

$$
g_{i} \sim \operatorname{Binomial}\left(2, f_{T}(i)\right)
$$

Under neutrality, we set $f_{T}(i)$ in (9) to:

$$
f_{T}^{N}(i)=\sum_{k=1}^{K}\left[\propto_{k}(i) \mathrm{f}_{\mathrm{k}}\right]
$$

815

816 For the alternative case of selection along the branches separating source $A$ and its sampled surrogate $A^{*}$, we generalize equation (8) and replace $f_{T}(i)$ in (9) with:

818

$$
f_{T}^{A}\left(i \mid s_{A}\right)=\left[\sum_{k !=A}^{K} \alpha_{A}(i) f_{k}\right]+\alpha_{A}(i)\left[\frac{\left(1+s_{A}\right) f_{A}}{1+s_{\mathrm{A}} f_{A}}\right]
$$


In practice, we fix $\propto_{A}(i)$ to be the proportion of DNA that each target individual $i$ matches to surrogate $k$ as inferred by ADMIXTURE. We define:

$$
\mathrm{L}^{\mathrm{P}}(s) \equiv \prod_{i=1}^{M}\left[f_{T}^{P}(i \mid s)^{g_{i}}\left(1-f_{T}^{P}(i \mid s)\right)^{2-g_{i}}\right]
$$

823

$$
L^{A}\left(s_{A}\right) \equiv \prod_{i=1}^{M}\left[f_{T}^{A}\left(i \mid s_{A}\right)^{g_{i}}\left(1-f_{T}^{A}\left(i \mid s_{A}\right)\right)^{2-g_{i}}\right]
$$

$$
\text { again finding } \hat{s}_{A} \text {, as the MLE for } s_{A} \text {. }
$$

We note that $\left[2-2 \log \left(L^{P}(\hat{s})\right]\right.$ and $\left[2-2 \log \left(L^{A}\left(\hat{S}_{A}\right)\right)\right]$ are analogous to AIC values for these respective models. Following AIC theory, we calculate:

$$
I=\frac{\min \left[L^{P}(\hat{s}), L^{A}\left(\hat{s}_{A}\right)\right]}{\max \left[L^{P}(\hat{s}), L^{A}\left(\hat{s}_{A}\right)\right]} \leq 1,
$$

where, relative to the model with higher likelihood out of (13) and (14), the model with smaller likelihood is $I$ times as probable to minimise the loss of information when used to represent the unknown true model (Akaike 1974). 


\section{Simulations}

\section{Estimating how well each surrogate reflects its corresponding true admixing source}

We aimed to generate simulations that mimic our real data. To do so, we first generate a measure of how well a sampled surrogate population $k$ reflects its corresponding true (unknown) source population. In particular, we estimate a drift parameter $d_{k}$ in the following manner. First, at each SNP $j$ we use nlminb in $\mathrm{R}$ to find the estimated values $\left\{\tilde{f}_{1}^{j}, \ldots, \tilde{f}_{K}^{j}\right\}$ for $\left\{f_{1^{*}}, \ldots, f_{K^{*}}\right\}$, respectively, that minimize:

$$
\sum_{i=1}^{M}\left(x_{i}^{j}-\sum_{k=1}^{K} \alpha_{k}(i) f_{k^{*}}\right)^{2}
$$

Where $x_{i}^{j} \in\{0,1,2\}$ is the allele count for the admixed target individual $i \in[1, \ldots, M]$ at the SNP and each $\tilde{f}_{k}^{j} \in[0,1]$. Then, for each source $k$, with observed allele counts $G_{k}^{j}$ and total counts $M_{k}^{j}$ at SNP $j$ in the surrogate population, following Balding-Nichols (Balding and Nichols 1995) we assume:

$$
G_{k}^{j} \text { Beta } \sim \text { Binomial }\left(M_{k}^{j} \frac{d_{k}}{1-d_{k}} \tilde{f}_{k}^{j}, \frac{d_{k}}{1-d_{k}}\left(1-\tilde{f}_{k}^{j}\right)\right)
$$

We then used the 'Nelder-Mead' algorithm in the optim function in $\mathrm{R}$ to find the $d_{k} \in[0,1]$ that maximized the product of (17) across all SNPs. This gave the values reported in Table 1.

Large estimated $d_{k}(>0.1)$ correspond to cases where there is little admixture from that source in our sampled individuals from that country, i.e. for African admixture in most countries and Native American admixture in Brazil. As values inferred using such little data are presumably unreliable, we cap them at 0.05 for the simulations below. While these values are a guide, in practice we adjusted these values by a multiple of 2-7 to generate neutral simulations that had the same inferred drift $\widehat{D}$, described in section 'Testing for evidence of selection at a SNP', as that observed in the real data. 


\begin{tabular}{|l|l|l|l|}
\hline Target & Native American & European & African \\
\hline Brazil & 0.173 & 0.007 & 0.102 \\
Chile & 0.02 & 0.011 & 0.226 \\
Colombia & 0.044 & 0.012 & 0.044 \\
Mexico & 0.024 & 0.007 & 0.223 \\
Peru & 0.015 & 0.009 & 0.119 \\
\hline
\end{tabular}

1. For each source $k$, at each SNP we sample starting allele frequencies $f_{k^{*}}$ from a $\operatorname{Beta}\left(\frac{d_{k}}{1-d_{k}} f_{k}, \frac{d_{k}}{1-d_{k}}\left(1-f_{k}\right)\right)$, where $f_{k}$ is the sampled frequency of the respective surrogate population and $d_{k}$ are defined in Table 1 (but capped at 0.05).

2. We randomly select SNPs to undergo selection. If selection is occurring in source population $k$ prior to admixture, we randomly sample from among SNPs for which $f_{k^{*}}<$ 0.5. If selection is occurring post-admixture, we instead randomly sample from among SNPs for which $\sum_{i=1}^{M}\left(\sum_{k=1}^{K} f_{k^{*}} \propto_{k}(i)\right) / M<0.5$.

3. We randomly select neutral SNPs from among all remaining SNPs, i.e., those not among the SNPs chosen in (2), in the real data.

4. To simulate selection:

- If selection is occurring prior to admixture, we simulate selection in the relevant source population for $g$ generations under a specified model of selection (additive, dominant, multiplicative, recessive) using Wright-Fisher with a population size of $N_{e}$ indiviuals.

- If selection is occurring after admixture, we simulate selection separately in each of the source populations for $g$ generations, under a specified model of selection using Wright-Fisher with a population size of $N_{e}$ individuals per population. 
5. At each SNP, we sample allele counts for each individual $i$ from a Binomial $\left(2, p_{i}\right)$ with $p_{i}=\sum_{k=1}^{K}\left[f_{k}^{g} \propto_{k}(i)\right]$, where:

- $f_{k}^{g}=\mathrm{f}_{\mathrm{k}^{*}}$ for neutral SNPs

- $f_{k}^{g}=f_{k^{*}}$ at selected SNPs for source populations $k$ not undergoing selection (i.e., in cases where selection is pre-admixture)

- $f_{k}^{g}$ is the sampled final frequencies in step (4) after $g$ generations, at selected SNPs for source population $k$ undergoing selection

We then analyse data from the simulated target population individuals using the real sampled data from the surrogate populations. For simulations here, we use $N_{e}=10000$ for the African,

Our procedure in steps (4)-(5) to simulate selection and admixture ensures the admixed individuals have variable admixture proportions while remaining computationally tractable. An alternative to this would be to generate $M$ admixed populations using observed $f_{k}$ values, with the admixture proportions for population $i$ equal to $\alpha_{1}(i), \ldots, \alpha_{K}(i)$, and then simulate each admixed population for $g$ generations using Wright-Fisher, either with or without selection. Such simulations would match the approach used by our model to classify selection as type (i) or type (ii) (Section

'Determining whether selection occurred pre- or post-admixture'). However, we chose the above for reasons of computational efficiency, as we have many individuals (i.e., $M>1000$ ). Note also that our selection test (Section 'Determining whether selection occurred pre- or post-admixture') is different from this simulation procedure, in that our test models the combined allele frequency across all admixed individuals, using the mean admixture contributions across target individuals to calculate the expected frequency. This may explain why our model exhibits an excess of SNPs with small $P$-values even when simulating no selection. This is despite using all SNPs to infer our model's variance parameter, which is designed to make more SNPs fit the model (likely explaining the excess of high $P$-values we also see, e.g., in supplementary fig. S14). While including this variance parameter does somewhat control $P$-values by e.g., giving a median $P$-value near 0.5 , as

\section{Local ancestry analysis}

935 Local ancestry assignment was conducted using the HMM approach implemented in ELAI (Guan 
2014). The phased genotype data needed as input was obtained by using SHAPEIT2 (Delaneau et al. 2012) with default parameter settings. Genetic distances were obtained from the HapMap Phase II genetic map build GRCh37 (Gibbs et al. 2003). As reference continental panels, we used the same Native American, European, and African individuals as in our AdaptMix analysis. ELAI was run setting the admixture generation parameter to 20 , and with 20 rounds of EM iterations. To obtain local ancestry assignment probabilities, we conducted 10 independent runs and averaged probabilities across all runs as recommended in the ELAI manual. To test for local ancestry deviations we estimated $Z$-scores for each ancestry across each locus, and obtained the corresponding one-sided $P$-values testing for a positive deviation.

\section{Population Branch Statistic (PBS) analysis}

947 We first selected Latin American individuals carrying a specific Native American ancestry component based on the inferred Native American ancestry proportions previously estimated by Chacon-Duque et al 2018 in the CANDELA sample. Specifically, for each Native American ancestry component, we selected CANDELA individuals with $>10 \%$ inferred ancestry from that particular Native American ancestry component, and with $<1 \%$ combined inferred ancestry combined across all other Native American components. Thus, each group of admixed Latin Americans was composed primarily of Native American ancestry from a particular Native American component, plus European and African ancestry. We then estimated allele frequencies for each Native American component by considering only alleles (i.e. haplotypes) that were considered of Native American origin with local-ancestry posterior probability $>0.9$. We only computed allele frequencies for a Native American component if all SNPs genome-wide had $>100$ alleles (haplotypes) assigned to Native American origin. This resulted in allele frequency estimates for six Native American components, including 'Quechua', 'Andes Piedmont', 'Chibcha Paez', 'Nahual', 'South Mexico', and 'Mapuche' ancestral components (see Chacon-Duque et al. (2018) for a detail description of the inferred components). Pairwise FST were then estimated using Hudson's estimator as in equation 9 of Bhatia et al. (2013). The branch length $(T)$ between two populations was 963 computed as $T=-\log _{10}\left(1-F_{S T}\right)$ (Cavalli-Sforza 1969). The Population Branch Statistic (PBS) 964 (Yi et al. 2010) combines the pairwise branch lengths between three populations, which was computed as:

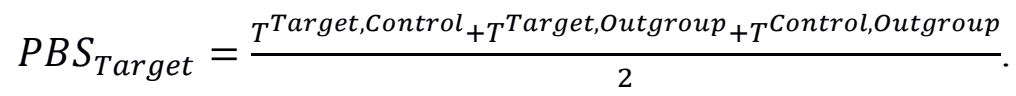

PBS values were computed for each Native American component, using all possible pairwise combinations of the other Native components as the control and outgroup populations. The rationale 
971 (i.e. that likely occurred after the divergence between Native American lineages). For some of our 972 analysis we also used the CHB population from the 1000 Genomes Project, the European reference 973 population, or the African reference population, as control and outgroup populations.

\section{Summary statistics for GWAS and eQTL data}

976 To assess the biological consequence of selected variants, we queried summary statistics from 977 GWASs of relevant phenotypes, and gene-expression data (i.e expression quantitative locus [eQTL] 978 studies) from relevant cell or tissues. For our GWAS query, we retrieved data from immune and 979 metabolic-related phenotypes, as these traits are known to have been subjected to strong selective 980 pressures across several human groups (Fan et al. 2016). Immune-related phenotypes included (i) 981 total white cell count, neutrophil count, lymphocyte count, monocyte count, basophil count, and 982 eosinophil count from the Chen et al. (2020) GWAS study conducted across five continental 983 ancestry groups. Metabolic-related phenotypes included body mass index (BMI), body fat 984 percentage, type II diabetes status, hip circumference, waist circumference, HDL levels, LDL 985 levels, cholesterol levels, and triglycerides levels (Loh et al. 2018). Summary statistics from these 986 GWAS analyses were based on the UK BioBank cohort available at: http://www.nealelab.is/uk987 biobank. For our eQTL query, we retrieved cis-associations summary statistics of 15 human 988 immune cell types from the DICE (Database of Immune Cell Expression, Expression quantitative 989 trait loci [eQTLs] and Epigenomics) project (Schmiedel et al. 2018), available at: https://dice990 database.org/downloads. We also retrieved cis-association summary statistics from adipose 991 (subcutaneous, and visceral omentum), muscle (skeletal), and liver tissue from the GTEx Project v7 992 (Lonsdale et al. 2013) available at: https://gtexportal.org/home/datasets.

\section{Acknowledgements}

We thank the volunteers for their enthusiastic support for this research. We also thank Alvaro Alvarado, Mónica Ballesteros Romero, Ricardo Cebrecos, Miguel Ángel Contreras Sieck, Francisco de Ávila Becerril, Joyce De la Piedra, María Teresa Del Solar, Paola Everardo Martínez, William Flores, Martha Granados Riveros, Rosilene Paim, Ricardo Gunski, Sergeant João Felisberto Menezes Cavalheiro, Major Eugênio Correa de Souza Junior, Wendy Hart, Ilich Jafet Moreno,

1001 Paola León-Mimila, Francisco Quispealaya, Diana Rogel Diaz, Ruth Rojas, and Vanessa Sarabia, 1002 for assistance with volunteer recruitment, sample processing and data entry. We also thank Francois 1003 Balloux, Aida Andres, Mark McCarthy, and Etienne Patin for helpful discussion and critical 1004 comments on earlier versions of the manuscript. We are very grateful to the institutions that allowed 1005 the use of their facilities for the assessment of volunteers, including: Escuela Nacional de 
Antropología e Historia and Universidad Nacional Autónoma de México (México); Universidade Federal do Rio Grande do Sul (Brazil); $13^{\circ}$ Companhia de Comunicações Mecanizada do Exército Brasileiro (Brazil); Pontificia Universidad Católica del Perú, Universidad de Lima and Universidad Nacional Mayor de San Marcos (Perú). Work leading to this publication received funded from: the National Natural Science Foundation of China (\#31771393 to ARL), the Scientific and Technology 1011 Committee of Shanghai Municipality (18490750300 to ARL), Ministry of Science and Technology 1012 of China (2020YFE0201600 to ARL), Shanghai Municipal Science and Technology Major Project 1013 (2017SHZDZX01 to ARL) and the 111 Project (B13016 to ARL), the Leverhulme Trust (F/07 1014 134/DF to ARL), BBSRC (BB/I021213/1 to ARL), the Excellence Initiative of Aix-Marseille 1015 University - A*MIDEX (a French "Investissements d'Avenir" programme to ARL), Wellcome 1016 Trust/Royal Society (098386/Z/12/Z to GH), the National Institute for Health Research University 1017 College London Hospitals Biomedical Research Centre, BBSRC (BB/R01356X/1), Universidad de 1018 Antioquia (CODI sostenibilidad de grupos 2013- 2014 and MASO 2013-2014), Conselho Nacional 1019 de Desenvolvimento Científico e Tecnológico, Fundação de Amparo à Pesquisa do Estado do Rio 1020 Grande do Sul (Apoio a Núcleos de Excelência Program) and Fundação de Aperfeiçoamento de 1021 Pessoal de Nível Superior. JM-R was supported by a doctoral scholarship from CONCYTEC-PERU (224-2014-FONDECYT).

\section{Data availability}

1025 1026 1027 1028 1029 1030 1031 1032 1033

1034 Scripts for selection analyses will be uploaded to a software developer public repository upon 1035 publication. The current version of AdaptMix presented in this study is available upon request from 1036 g.hellenthal@ucl.ac.uk.

This project only analyses data that has been previously reported in other publications. Raw genotype data for reference populations can be accessed as described previously (The 1000 Genomes Project Consortium 2015; Chacon-Duque et al. 2018). Raw genotype data from CANDELA cannot be made available due to restrictions imposed by ethical approval. Summary statistics from the selection analysis will be deposited in a public repository upon publication.

\section{Software availability}


1038

1039

1040

1041

1042

1043

1044

1045

1046

1047

1048

1049

1050

1051

1052

1053

1054

1055

1056

1057

1058

1059

1060

1061

1062

1063

1064

1065

1066

1067

1068

1069

1070

1071

\section{Main Figure legends}

Fig. 1. Schematic and intuition of the AdaptMix model. (a) For each CANDELA individual (columns), ADMIXTURE-inferred proportions of ancestry related to Native American, European, and African reference individuals. (b) Assuming only two admixing sources in this illustration for simplicity, the model assumes ancestral populations $\left(K_{1}^{\prime}\right.$ and $\left.K_{2}^{\prime}\right)$ contribute ancestry proportions $\alpha_{K_{1}}$ and $\alpha_{K_{2}}$, respectively, to an admixed population $\left(X^{\prime}\right)$ that is ancestral to the tested population $(X)$. Assuming neutrality, the expected allele frequency $\left(p_{0}\right)$ of $X^{\prime}$ is estimated using these proportions and the allele frequencies surrogate populations $K_{1}$ and $K_{2}$ related to $K_{1}^{\prime}$ and $K_{2}^{\prime}$, respectively. The sampled allele frequency $(p)$ of $X$ is compared to $p_{0}$, with large deviations indicative of selection (shown with an asterisk in the distribution). (c and d) The relationship between $p_{0}$, the expected allele frequency in the admixed population under neutrality or selection, and $\alpha_{K_{2}}$, the ancestry proportion contributed from ancestral population $K_{2}^{\prime}$. If selection occurred prior to admixture during the split between populations $K_{2}^{\prime}$ and its surrogate $K_{2}$ (i.e. along the blue branch in [a]), this relationship increases linearly (blue lines), becoming more differentiated from neutrality (grey line) as the admixture from $K_{2}^{\prime}$ increases. In contrast, under selection post-admixture (i.e. along the purple branch in a]), the expected allele frequency (purple lines) can deviate from neutrality even when the admixture from $K_{2}^{\prime}$ is near 0 . The difference between the post-admixture and pre-admixture lines is more clear when allele frequencies in populations $K_{1}$ and $K_{2}$ are similar (top plot). Solid blue and red lines indicate the allele frequencies in the surrogate populations $K_{1}$ and $K_{2}$, which are used to calculate $p_{0}$.

\section{Fig 2. Performance of AdaptMix to detect and classify selection in simulated Latin American} populations. (a) Power to detect selection post-admixture, selection in Native Americans, or selection in Europeans in simulated populations mimicking the Latin American cohorts. Power is based on a $P$-value cutoff that resulted in a false-positive rate of $5 \times 10^{-5}$ in neutral simulations. The power estimated for a given selection coefficient is based on combining simulations using four different modes of selection (additive, dominant, multiplicative, recessive) over 12 generations for the post-admixture simulations, over 50 generations for the selection in Native American simulations, and over 25 generations for the selection in European simulations. Each simulation for a given combination of parameters consisted of 10,000 advantageous SNPs with a pre-selection minor allele frequency lower than 0.5. (b) The proportion of significant SNPs from (a) that were assigned to the correct simulated scenario of (left-to-right) post-admixture selection or selection in Native Americans or Europeans (using a likelihood ratio $>1,000$ to make a call; otherwise 'Unclassified'). Rows give the true selection coefficient (legend at right), and the heatmap values 
1072 give the classification rate. Rows with N.A. shows instances with less than 50 selected SNPs for 1073 which the classification rate is not shown.

1074

1075

1076

1077

1078

1079

1080

1081

1082

1083

1084

1085

1086

1087

1088

1089

1090

1091

1092

1093

1094

1095

1096

1097

1098

1099

1100

1101

1102

1103

1104

1105

1106

Fig. 3. Genome-wide selection scan in five Latin American cohorts. Manhattan plot showing the genomic regions identified as selected via AdaptMix in each Latin American cohort. The dashed horizontal lines indicate the $P$-values cutoffs corresponding to a false-positive rate of $5 \times 10^{-5}$ based on neutral simulations. Different shapes represent the most likely selection model. Names of genes associated with significant SNPs are shown.

Fig 4. Regional selection plot at the HLA region in five Latin American cohorts. The top plot shows the $-\log _{10}(P$-values) of SNPs from AdaptMix, the middle plot shows $Z$-score values based on African local ancestry deviations, and the bottom plot shows genes in the region shaded in grey. Genomic coordinates are in $\mathrm{Mb}$ (build hg19 as reference) and genes shown include transcripts.

Fig. 5. Genetic loci with signals of selection at immune-related genes. (a), (b) and (c) Regional selection plot at three candidate regions of selection encompassing two immune-related genes in the Chilean and one immune-related gene in the Peruvian cohort, respectively. Each plot is composed of four panels (rows), consisting of $-\log _{10}(P$-values) of SNPs: (row 1) from AdaptMix; (row 2) associated with immune-related cell counts via GWAS (Chen et al 2020); (row 3) associated (as expression quantitative trait loci [eQTLs]) with expression of genes CD101, PTPN2 and MIF for (a)-(c), respectively (Schmiedel et al. 2018); with (row 4) depicting genes in the region (in Mb, build hg19 as reference. Horizontal dashed lines give significance thresholds of (row 1) $P$ value $=1 \times 10^{-5}$ based on neutral simulations (row 2) $P$-value $=1 \times 10^{-5}$ (blue line) and $P$ value $=5 \times 10^{-8}$ (red line), and (row 3) $P$-value $=1 \times 10^{-4}$. (d), (e) and (f) Derived allele frequency (DAF) in admixed Latin Americans (white circles) stratified by proportion of inferred Native American ancestry, for the SNPs highlighted (vertical dashed line) in top row panels. The sizes of the circles are proportional to the number of individuals in that particular bin. Lines give expected DAF under neutrality (grey), post-admixture selection (brown) or selection in the Native source (black). Horizontal dashed red, blue, and green lines depict DAF for surrogates to Native American, European, and African sources, respectively.

Fig. 6. Genetic loci with signals of selection at metabolic-related genes. (a) and (b) Regional selection plot at two candidate regions of selection encompassing metabolic-related genes in the Mexican and Peruvian cohorts, respectively. Each plot is composed of four panels consisting of $-\log _{10}(P$-values) of SNPs: (row 1) from AdaptMix; (row 2) from the UK Biobank GWAS; (row 3) 
1107 associated (as eQTLs) with expression of BRINP3 and HKDC1 for (a)-(b), respectively, (GTEx

1108 eQTL study); with (row 4) depicting genes in the region (in Mb, build hg19 as reference).

1109 Horizontal dashed lines give significance thresholds of (row 1) $P$-value $=1 \times 10^{-5}$ based on neutral 1110 simulations (row 2) $P$-value $=1 \times 10^{-5}$ (blue line) and $P$-value $=5 \times 10^{-8}$ (red line), and (row 3) $P$ 1111 value $=1 \times 10^{-4}$. (c) and (d) Derived allele frequency (DAF) in admixed Latin Americans (white 1112 circles) stratified by proportion of inferred Native American ancestry, for the SNPs highlighted 1113 (vertical dashed line) in top row panels. The sizes of the circles are proportional to the number of 1114 individuals in that particular bin. Lines give expected DAF under neutrality (grey), post-admixture 1115 selection (brown) or selection in the Native American source (black). Horizontal dashed red, blue, 1116 and green lines depict DAF for surrogates to Native American, European, and African sources, 1117 respectively. 


\section{References}

1120 Acuña-Alonzo V, Flores-Dorantes T, Kruit JK, Villarreal-Molina T, Arellano-Campos O,

1121 Hünemeier T, Moreno-Estrada A, Ortiz-López MG, Villamil-Ramírez H, León-Mimila P. 2010. A

1122 functional ABCA1 gene variant is associated with low HDL-cholesterol levels and shows evidence

1123 of positive selection in Native Americans. Human molecular genetics 19:2877-2885.

1124

1125

1126

1127

1128

1129

1130

1131

1132

1133

1134

1135

1136

1137

1138

1139

1140 Akaike H. 1974. A new look at the statistical model identification. IEEE transactions on automatic control 19:716-723.

Alexander DH, Novembre J, Lange K. 2009. Fast model-based estimation of ancestry in unrelated individuals. Genome Res 19:1655-1664.

Amorim CE, Nunes K, Meyer D, Comas D, Bortolini MC, Salzano FM, Hunemeier T. 2017. Genetic signature of natural selection in first Americans. Proc Natl Acad Sci U S A 114:2195-2199. Avila-Arcos MC, McManus KF, Sandoval K, Rodriguez-Rodriguez JE, Villa-Islas V, Martin AR, Luisi P, Penaloza-Espinosa RI, Eng C, Huntsman S, et al. 2020. Population History and Gene Divergence in Native Mexicans Inferred from 76 Human Exomes. Mol Biol Evol 37:994-1006. Badillo Rivera KM, Nieves-Colón MA, Mendoza KS, Davalos VV, Lencinas LEE, Chen JW, Zhang ET, Sockell A, Tello PO, Hurtado GM. 2021. Clotting factor genes are associated with preeclampsia in high altitude pregnant women in the Peruvian Andes. medRxiv.

Balding DJ, Nichols RA. 1995. A method for quantifying differentiation between populations at multi-allelic loci and its implications for investigating identity and paternity. Genetica 96:3-12. Basu A, Tang H, Zhu X, Gu CC, Hanis C, Boerwinkle E, Risch N. 2008. Genome-wide distribution of ancestry in Mexican Americans. Hum Genet 124:207-214.

Bersaglieri T, Sabeti PC, Patterson N, Vanderploeg T, Schaffner SF, Drake JA, Rhodes M, Reich DE, Hirschhorn JN. 2004. Genetic signatures of strong recent positive selection at the lactase gene. Am J Hum Genet 74:1111-1120.

Bhatia G, Patterson N, Sankararaman S, Price AL. 2013. Estimating and interpreting FST: the impact of rare variants. Genome research 23:1514-1521.

Bhatia G, Tandon A, Patterson N, Aldrich MC, Ambrosone CB, Amos C, Bandera EV, Berndt SI, Bernstein L, Blot WJ. 2014. Genome-wide scan of 29,141 African Americans finds no evidence of directional selection since admixture. The American Journal of Human Genetics 95:437-444. Borrego F. 2013. The CD300 molecules: an emerging family of regulators of the immune system. Blood, The Journal of the American Society of Hematology 121:1951-1960.

Bouloc A, Bagot M, Delaire S, Bensussan A, Boumsell L. 2000. Triggering CD101 molecule on human cutaneous dendritic cells inhibits $\mathrm{T}$ cell proliferation via IL-10 production. European journal of immunology 30:3132-3139.

Calandra T, Roger T. 2003. Macrophage migration inhibitory factor: a regulator of innate immunity. Nature Reviews Immunology 3:791-800.

Cavalli-Sforza LL editor.; 1969.

Chacon-Duque JC, Adhikari K, Fuentes-Guajardo M, Mendoza-Revilla J, Acuna-Alonzo V, Barquera R, Quinto-Sanchez M, Gomez-Valdes J, Everardo Martinez P, Villamil-Ramirez H, et al. 2018. Latin Americans show wide-spread Converso ancestry and imprint of local Native ancestry on physical appearance. Nat Commun 9:5388.

Chang CC, Chow CC, Tellier LC, Vattikuti S, Purcell SM, Lee JJ. 2015. Second-generation PLINK: rising to the challenge of larger and richer datasets. Gigascience 4:s13742-13015-1004713748.

Chen M-H, Raffield LM, Mousas A, Sakaue S, Huffman JE, Moscati A, Trivedi B, Jiang T, Akbari P, Vuckovic D. 2020. Trans-ethnic and ancestry-specific blood-cell genetics in 746,667 individuals from 5 global populations. Cell 182:1198-1213. e1114.

Cheng JY, Stern AJ, Racimo F, Nielsen R. 2021. Detecting selection in multiple populations by modelling ancestral admixture components. Mol Biol Evol.

Consortium TGP. 2015. A global reference for human genetic variation. Nature 526:68.

Delaneau O, Marchini J, Zagury J-F. 2012. A linear complexity phasing method for thousands of genomes. Nature methods 9:179-181. 
1171 Deng L, Ruiz-Linares A, Xu S, Wang S. 2016. Ancestry variation and footprints of natural selection

1172

1173

1174

1175

1176

1177

1178

1179

1180

1181

1182

1183

1184

1185

1186

1187

1188

1189

1190

1191

1192

1193

1194

1195

1196

1197

1198

1199

1200

1201

1202

1203

1204

1205

1206

1207

1208

1209

1210

1211

1212

1213

1214

1215

1216

1217

1218

1219

1220

along the genome in Latin American populations. Sci Rep 6:21766.

Ettinger NA, Duggal P, Braz RF, Nascimento ET, Beaty TH, Jeronimo SM, Pearson RD, Blackwell JM, Moreno L, Wilson ME. 2009. Genetic admixture in Brazilians exposed to infection with Leishmania chagasi. Ann Hum Genet 73:304-313.

Fan S, Hansen ME, Lo Y, Tishkoff SA. 2016. Going global by adapting local: A review of recent human adaptation. Science 354:54-59.

Galinsky KJ, Bhatia G, Loh PR, Georgiev S, Mukherjee S, Patterson NJ, Price AL. 2016. Fast Principal-Component Analysis Reveals Convergent Evolution of ADH1B in Europe and East Asia. Am J Hum Genet 98:456-472.

Ghoussaini M, Mountjoy E, Carmona M, Peat G, Schmidt EM, Hercules A, Fumis L, Miranda A, Carvalho-Silva D, Buniello A. 2021. Open Targets Genetics: systematic identification of traitassociated genes using large-scale genetics and functional genomics. Nucleic acids research 49:D1311-D1320.

Gibbs RA, Belmont JW, Hardenbol P, Willis TD, Yu F, Yang H, Ch'ang L-Y, Huang W, Liu B, Shen Y. 2003. The international HapMap project.

Giri A, Hellwege JN, Keaton JM, Park J, Qiu C, Warren HR, Torstenson ES, Kovesdy CP, Sun YV, Wilson OD. 2019. Trans-ethnic association study of blood pressure determinants in over 750,000 individuals. Nature genetics 51:51-62.

Gu S, Li H, Pakstis AJ, Speed WC, Gurwitz D, Kidd JR, Kidd KK. 2018. Recent Selection on a Class I ADH Locus Distinguishes Southwest Asian Populations Including Ashkenazi Jews. Genes (Basel) 9.

Guan Y. 2014. Detecting structure of haplotypes and local ancestry. Genetics 196:625-642.

Guo C, Ludvik AE, Arlotto ME, Hayes MG, Armstrong LL, Scholtens DM, Brown CD, Newgard CB, Becker TC, Layden BT. 2015. Coordinated regulatory variation associated with gestational hyperglycaemia regulates expression of the novel hexokinase HKDC1. Nature communications 6:18.

Hamid I, Korunes KL, Beleza S, Goldberg A. 2021. Rapid adaptation to malaria facilitated by admixture in the human population of Cabo Verde. Elife 10.

Hancock AM, Witonsky DB, Ehler E, Alkorta-Aranburu G, Beall C, Gebremedhin A, Sukernik R, Utermann G, Pritchard J, Coop G. 2010. Human adaptations to diet, subsistence, and ecoregion are due to subtle shifts in allele frequency. Proceedings of the National Academy of Sciences 107:89248930.

Harris DN, Ruczinski I, Yanek LR, Becker LC, Becker DM, Guio H, Cui T, Chilton FH, Mathias RA, O'Connor TD. 2019. Evolution of hominin polyunsaturated fatty acid metabolism: from Africa to the New World. Genome biology and evolution 11:1417-1430.

Hayes MG, Urbanek M, Hivert M-F, Armstrong LL, Morrison J, Guo C, Lowe LP, Scheftner DA, Pluzhnikov A, Levine DM. 2013. Identification of HKDC1 and BACE2 as genes influencing glycemic traits during pregnancy through genome-wide association studies. Diabetes 62:3282-3291. Hellenthal G, Busby GBJ, Band G, Wilson JF, Capelli C, Falush D, Myers S. 2014. A genetic atlas of human admixture history. Science 343:747-751.

Hodgson JA, Pickrell JK, Pearson LN, Quillen EE, Prista A, Rocha J, Soodyall H, Shriver MD, Perry GH. 2014. Natural selection for the Duffy-null allele in the recently admixed people of Madagascar. Proc Biol Sci 281:20140930.

Hoffmann TJ, Ehret GB, Nandakumar P, Ranatunga D, Schaefer C, Kwok P-Y, Iribarren C, Chakravarti A, Risch N. 2017. Genome-wide association analyses using electronic health records identify new loci influencing blood pressure variation. Nature genetics 49:54-64.

Homburger JR, Moreno-Estrada A, Gignoux CR, Nelson D, Sanchez E, Ortiz-Tello P, Pons-Estel BA, Acevedo-Vasquez E, Miranda P, Langefeld CD, et al. 2015. Genomic Insights into the Ancestry and Demographic History of South America. PLoS Genet 11:e1005602. 
1221 Joffe GM, Esterlitz JR, Levine RJ, Clemens JD, Ewell MG, Sibai BM, Catalano PM. 1998. The

1222

1223

1224

1225

1226

1227

1228

1229

1230

1231

1232

1233

1234

1235

1236

1237

1238

1239

1240

1241

1242

1243

1244

1245

1246

1247

1248

1249

1250

1251

1252

1253

1254

1255

1256

1257

1258

1259

1260

1261

1262

1263

1264

1265

1266

1267

1268

1269

1270

1271

relationship between abnormal glucose tolerance and hypertensive disorders of pregnancy in

healthy nulliparous women. American journal of obstetrics and gynecology 179:1032-1037.

Kanthimathi S, Liju S, Laasya D, Anjana RM, Mohan V, Radha V. 2016. Hexokinase domain containing 1 (HKDC1) gene variants and their association with gestational diabetes mellitus in a south indian population. Annals of human genetics 80:241-245.

Karlsson EK, Kwiatkowski DP, Sabeti PC. 2014. Natural selection and infectious disease in human populations. Nature Reviews Genetics 15:379-393.

Kominsky DJ, Campbell EL, Colgan SP. 2010. Metabolic shifts in immunity and inflammation.

The Journal of Immunology 184:4062-4068.

Koscielny G, An P, Carvalho-Silva D, Cham JA, Fumis L, Gasparyan R, Hasan S, Karamanis N, Maguire M, Papa E. 2017. Open Targets: a platform for therapeutic target identification and validation. Nucleic acids research 45:D985-D994.

Lazaridis I, Patterson N, Mittnik A, Renaud G, Mallick S, Kirsanow K, Sudmant PH, Schraiber JG, Castellano S, Lipson M, et al. 2014. Ancient human genomes suggest three ancestral populations for present-day Europeans. Nature 513:409-413.

Lindo J, Haas R, Hofman C, Apata M, Moraga M, Verdugo RA, Watson JT, Llave CV, Witonsky D, Beall C. 2018. The genetic prehistory of the Andean highlands 7000 years BP though European contact. Science advances 4:eaau4921.

Loh PR, Kichaev G, Gazal S, Schoech AP, Price AL. 2018. Mixed-model association for biobankscale datasets. Nat Genet 50:906-908.

Long JC. 1991. The genetic structure of admixed populations. Genetics 127:417-428.

Lonsdale J, Thomas J, Salvatore M, Phillips R, Lo E, Shad S, Hasz R, Walters G, Garcia F, Young N. 2013. The genotype-tissue expression (GTEx) project. Nature genetics 45:580-585.

Ludvik AE, Pusec CM, Priyadarshini M, Angueira AR, Guo C, Lo A, Hershenhouse KS, Yang GY, Ding X, Reddy TE. 2016. HKDC1 is a novel hexokinase involved in whole-body glucose use. Endocrinology 157:3452-3461.

Luisi P, García A, Berros JM, Motti JM, Demarchi DA, Alfaro E, Aquilano E, Argüelles C, Avena S, Bailliet G. 2020. Fine-scale genomic analyses of admixed individuals reveal unrecognized genetic ancestry components in Argentina. PloS one 15:e0233808.

Lumeng CN, Saltiel AR. 2011. Inflammatory links between obesity and metabolic disease. The Journal of clinical investigation 121:2111-2117.

Mathieson I. 2020. Limited evidence for selection at the FADS locus in Native American populations. Molecular biology and evolution 37:2029-2033.

Mathieson I, Lazaridis I, Rohland N, Mallick S, Patterson N, Roodenberg SA, Harney E, Stewardson K, Fernandes D, Novak M, et al. 2015. Genome-wide patterns of selection in 230 ancient Eurasians. Nature 528:499-503.

Moreno-Estrada A, Gignoux CR, Fernandez-Lopez JC, Zakharia F, Sikora M, Contreras AV, Acuna-Alonzo V, Sandoval K, Eng C, Romero-Hidalgo S, et al. 2014. Human genetics. The genetics of Mexico recapitulates Native American substructure and affects biomedical traits. Science 344:1280-1285.

Moreno-Estrada A, Gravel S, Zakharia F, McCauley JL, Byrnes JK, Gignoux CR, Ortiz-Tello PA, Martinez RJ, Hedges DJ, Morris RW, et al. 2013. Reconstructing the population genetic history of the Caribbean. PLoS Genet 9:e1003925.

Norris ET, Rishishwar L, Chande AT, Conley AB, Ye K, Valderrama-Aguirre A, Jordan IK. 2020. Admixture-enabled selection for rapid adaptive evolution in the Americas. Genome Biol 21:29.

Osuna-Ramos JF, Reyes-Ruiz JM, Del Ángel RM. 2018. The role of host cholesterol during flavivirus infection. Frontiers in cellular and infection microbiology 8:388.

Pasaniuc B, Sankararaman S, Torgerson DG, Gignoux C, Zaitlen N, Eng C, Rodriguez-Cintron W, Chapela R, Ford JG, Avila PC. 2013. Analysis of Latino populations from GALA and MEC studies reveals genomic loci with biased local ancestry estimation. Bioinformatics 29:1407-1415. 
1272 Patterson N, Moorjani P, Luo Y, Mallick S, Rohland N, Zhan Y, Genschoreck T, Webster T, Reich D. 2012. Ancient admixture in human history. Genetics 192:1065-1093.

Pickup J, Crook M. 1998. Is type II diabetes mellitus a disease of the innate immune system? Diabetologia 41:1241-1248. Pierron D, Heiske M, Razafindrazaka H, Pereda-Loth V, Sanchez J, Alva O, Arachiche A, Boland A, Olaso R, Deleuze JF, et al. 2018. Strong selection during the last millennium for African ancestry in the admixed population of Madagascar. Nat Commun 9:932.

Poulter M, Hollox E, Harvey CB, Mulcare C, Peuhkuri K, Kajander K, Sarner M, Korpela R, Swallow DM. 2003. The causal element for the lactase persistence/non-persistence polymorphism is located in a $1 \mathrm{Mb}$ region of linkage disequilibrium in Europeans. Ann Hum Genet 67:298-311. Pulit SL, Stoneman C, Morris AP, Wood AR, Glastonbury CA, Tyrrell J, Yengo L, Ferreira T, Marouli E, Ji Y. 2019. Meta-analysis of genome-wide association studies for body fat distribution in 694649 individuals of European ancestry. Human molecular genetics 28:166-174.

Racimo F, Gokhman D, Fumagalli M, Ko A, Hansen T, Moltke I, Albrechtsen A, Carmel L, Huerta-Sánchez E, Nielsen R. 2017. Archaic adaptive introgression in TBX15/WARS2. Molecular biology and evolution 34:509-524.

Reynolds AW, Mata-Miguez J, Miro-Herrans A, Briggs-Cloud M, Sylestine A, Barajas-Olmos F, Garcia-Ortiz H, Rzhetskaya M, Orozco L, Raff JA, et al. 2019. Comparing signals of natural selection between three Indigenous North American populations. Proc Natl Acad Sci U S A 116:9312-9317.

Rishishwar L, Conley AB, Wigington CH, Wang L, Valderrama-Aguirre A, Jordan IK. 2015. Ancestry, admixture and fitness in Colombian genomes. Sci Rep 5:12376.

Robbins GR, Wen H, Ting JP-Y. 2014. Inflammasomes and metabolic disorders: old genes in modern diseases. Molecular cell 54:297-308.

Ruiz-Linares A, Adhikari K, Acuna-Alonzo V, Quinto-Sanchez M, Jaramillo C, Arias W, Fuentes M, Pizarro M, Everardo P, de Avila F, et al. 2014. Admixture in Latin America: geographic structure, phenotypic diversity and self-perception of ancestry based on 7,342 individuals. PLoS Genet 10:e1004572. Rumold CU, Aldenderfer MS. 2016. Late Archaic-Early Formative period microbotanical evidence for potato at Jiskairumoko in the Titicaca Basin of southern Peru. Proceedings of the National Academy of Sciences 113:13672-13677.

Santoscoy-Ascencio G, Baños-Hernández CJ, Navarro-Zarza JE, Hernández-Bello J, Bucala R, López-Quintero A, Valdés-Alvarado E, Parra-Rojas I, Illades-Aguiar B, Muñoz-Valle JF. 2020. Macrophage migration inhibitory factor promoter polymorphisms are associated with disease activity in rheumatoid arthritis patients from Southern Mexico. Molecular genetics \& genomic medicine 8:e1037.

Schmiedel BJ, Singh D, Madrigal A, Valdovino-Gonzalez AG, White BM, Zapardiel-Gonzalo J, Ha B, Altay G, Greenbaum JA, McVicker G. 2018. Impact of genetic polymorphisms on human immune cell gene expression. Cell 175:1701-1715. e1716.

Shuai K, Liu B. 2003. Regulation of JAK-STAT signalling in the immune system. Nature Reviews Immunology 3:900-911.

Sibai BM. 2003. Diagnosis and management of gestational hypertension and preeclampsia. Obstetrics \& Gynecology 102:181-192.

Sirugo G, Williams SM, Tishkoff SA. 2019. The Missing Diversity in Human Genetic Studies. Cell 177:1080.

Soares LR, Tsavaler L, Rivas A, Engleman EG. 1998. V7 (CD101) ligation inhibits TCR/CD3induced IL-2 production by blocking $\mathrm{Ca} 2+$ flux and nuclear factor of activated $\mathrm{T}$ cell nuclear translocation. The Journal of Immunology 161:209-217.

Tan Y-X, Hu S-M, You Y-P, Yang G-L, Wang W. 2019. Replication of previous genome-wide association studies of HKDC1, BACE2, SLC16A11 and TMEM163 SNPs in a gestational diabetes mellitus case-control sample from Han Chinese population. Diabetes, metabolic syndrome and obesity: targets and therapy 12:983. 
Tang H, Choudhry S, Mei R, Morgan M, Rodriguez-Cintron W, Burchard EG, Risch NJ. 2007. Recent genetic selection in the ancestral admixture of Puerto Ricans. Am J Hum Genet 81:626-633. Van Dijk M, Mulders J, Poutsma A, Könst AA, Lachmeijer AM, Dekker GA, Blankenstein MA, Oudejans CB. 2005. Maternal segregation of the Dutch preeclampsia locus at 10q22 with a new member of the winged helix gene family. Nature genetics 37:514-519. van Dijk M, Oudejans C. 2011. STOX1: key player in trophoblast dysfunction underlying early onset preeclampsia with growth retardation. Journal of pregnancy 2011.

Vicente M, Priehodova E, Diallo I, Podgorna E, Poloni ES, Cerny V, Schlebusch CM. 2019. Population history and genetic adaptation of the Fulani nomads: inferences from genome-wide data and the lactase persistence trait. BMC Genomics 20:915.

Vicuna L, Klimenkova O, Norambuena T, Martinez FI, Fernandez MI, Shchur V, Eyheramendy S. 2020. Postadmixture Selection on Chileans Targets Haplotype Involved in Pigmentation, Thermogenesis and Immune Defense against Pathogens. Genome Biol Evol 12:1459-1470. Villarreal-Molina MT, Flores-Dorantes MT, Arellano-Campos O, Villalobos-Comparan M, Rodríguez-Cruz M, Miliar-García A, Huertas-Vazquez A, Menjivar M, Romero-Hidalgo S, Wacher NH. 2008. Association of the ATP-binding cassette transporter A1 R230C variant with early-onset type 2 diabetes in a Mexican population. Diabetes 57:509-513.

Wang S, Lewis CM, Jakobsson M, Ramachandran S, Ray N, Bedoya G, Rojas W, Parra MV, Molina JA, Gallo C, et al. 2007. Genetic variation and population structure in native Americans. PLoS Genet 3:e185.

Warren HR, Evangelou E, Cabrera CP, Gao H, Ren M, Mifsud B, Ntalla I, Surendran P, Liu C, Cook JP. 2017. Genome-wide association analysis identifies novel blood pressure loci and offers biological insights into cardiovascular risk. Nature genetics 49:403-415.

Warrington NM, Beaumont RN, Horikoshi M, Day FR, Helgeland Ø, Laurin C, Bacelis J, Peng S, Hao K, Feenstra B. 2019. Maternal and fetal genetic effects on birth weight and their relevance to cardio-metabolic risk factors. Nature genetics 51:804-814.

Weissgerber TL, Mudd LM. 2015. Preeclampsia and diabetes. Current diabetes reports 15:1-10. Yi X, Liang Y, Huerta-Sanchez E, Jin X, Cuo ZXP, Pool JE, Xu X, Jiang H, Vinckenbosch N, Korneliussen TS. 2010. Sequencing of 50 human exomes reveals adaptation to high altitude. Science 329:75-78.

Zamudio S. 2007. High-altitude hypoxia and preeclampsia. Frontiers in bioscience: a journal and virtual library 12:2967.

Zhou Q, Zhao L, Guan Y. 2016. Strong selection at MHC in Mexicans since admixture. PLoS genetics 12:e1005847.

Zhu Z, Guo Y, Shi H, Liu C-L, Panganiban RA, Chung W, O'Connor LJ, Himes BE, Gazal S, Hasegawa K. 2020. Shared genetic and experimental links between obesity-related traits and asthma subtypes in UK Biobank. Journal of Allergy and Clinical Immunology 145:537-549. 
a 1.
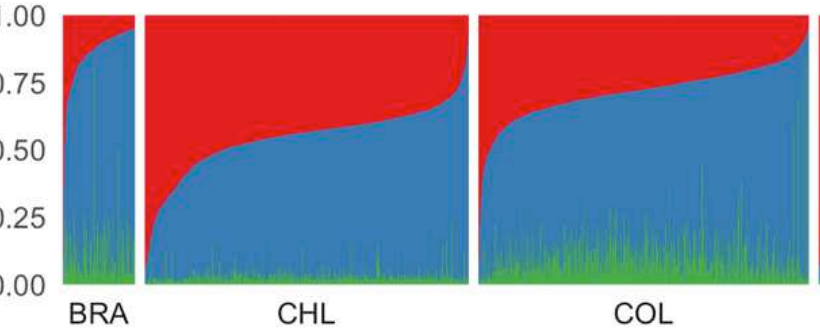

$\mathrm{COL}$

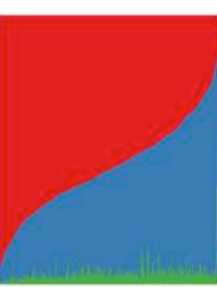

MEX

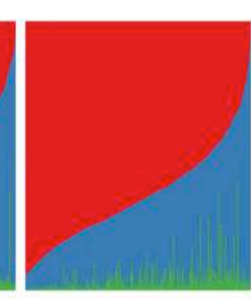

PER
Native American $\rightarrow a_{K 1}$

European $\rightarrow a_{K 2}$

African
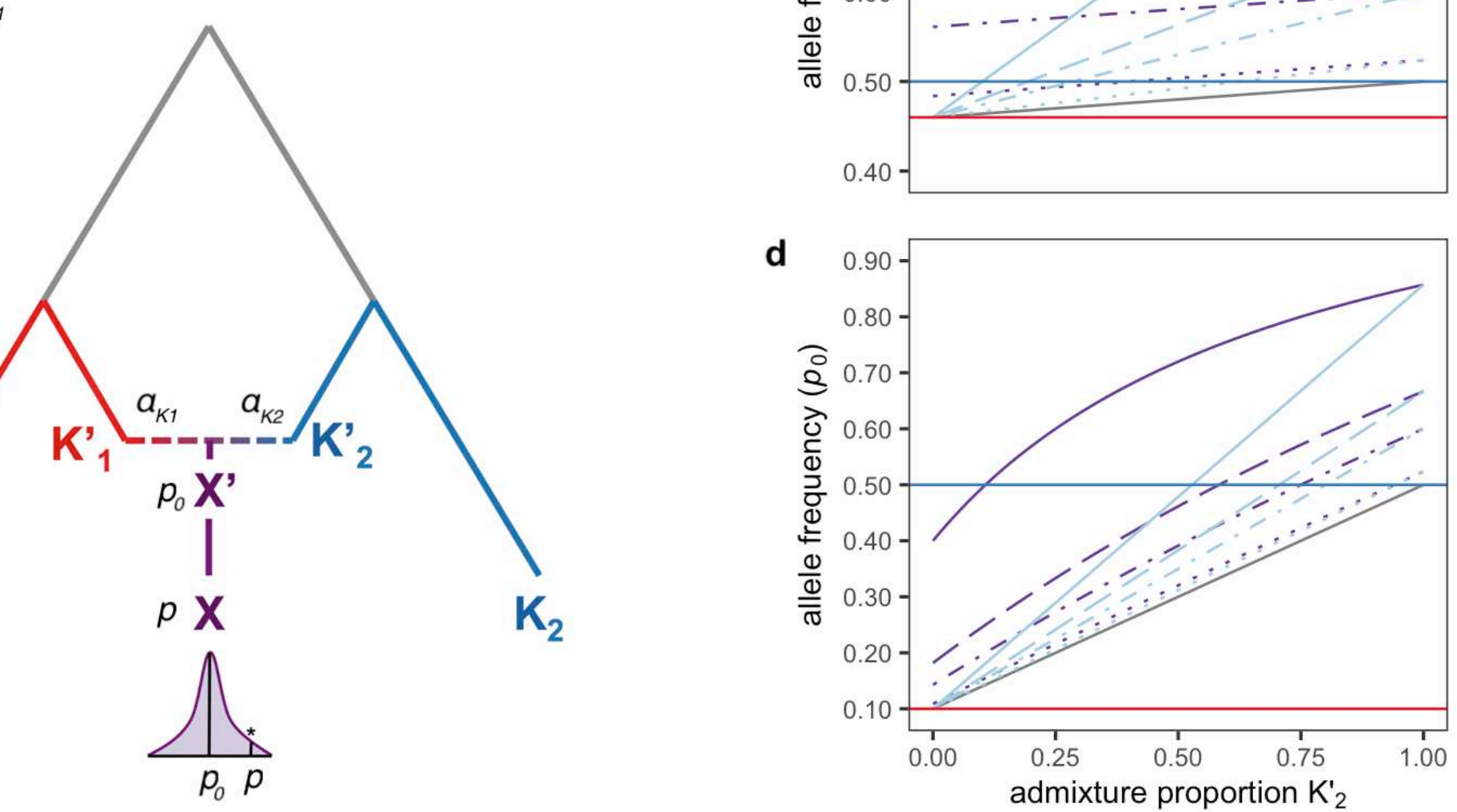

d

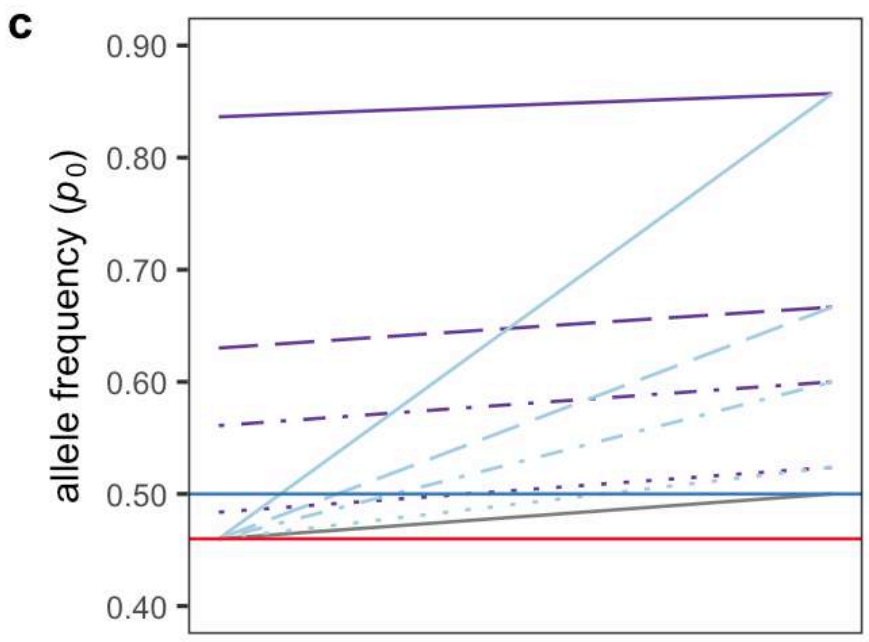

Model

- Neutral

- Post-admixture ( $\mathrm{s}=0.1)$

- Post-admixture $(\mathrm{s}=0.5)$

- Post-admixture $(\mathrm{s}=1)$

- Post-admixture ( $\mathrm{s}=5$ )

Selection in $\mathrm{K}_{2}^{\prime} / \mathrm{K}_{2}(\mathrm{~s}=0.1)$

Selection in $\mathrm{K}_{2}^{\prime} / \mathrm{K}_{2}(\mathrm{~s}=0.5)$

- Selection in $\mathrm{K}_{2} / \mathrm{K}_{2}(\mathrm{~s}=1)$

- Selection in $\mathrm{K}_{2}^{\prime} / \mathrm{K}_{2}(\mathrm{~s}=5)$ 


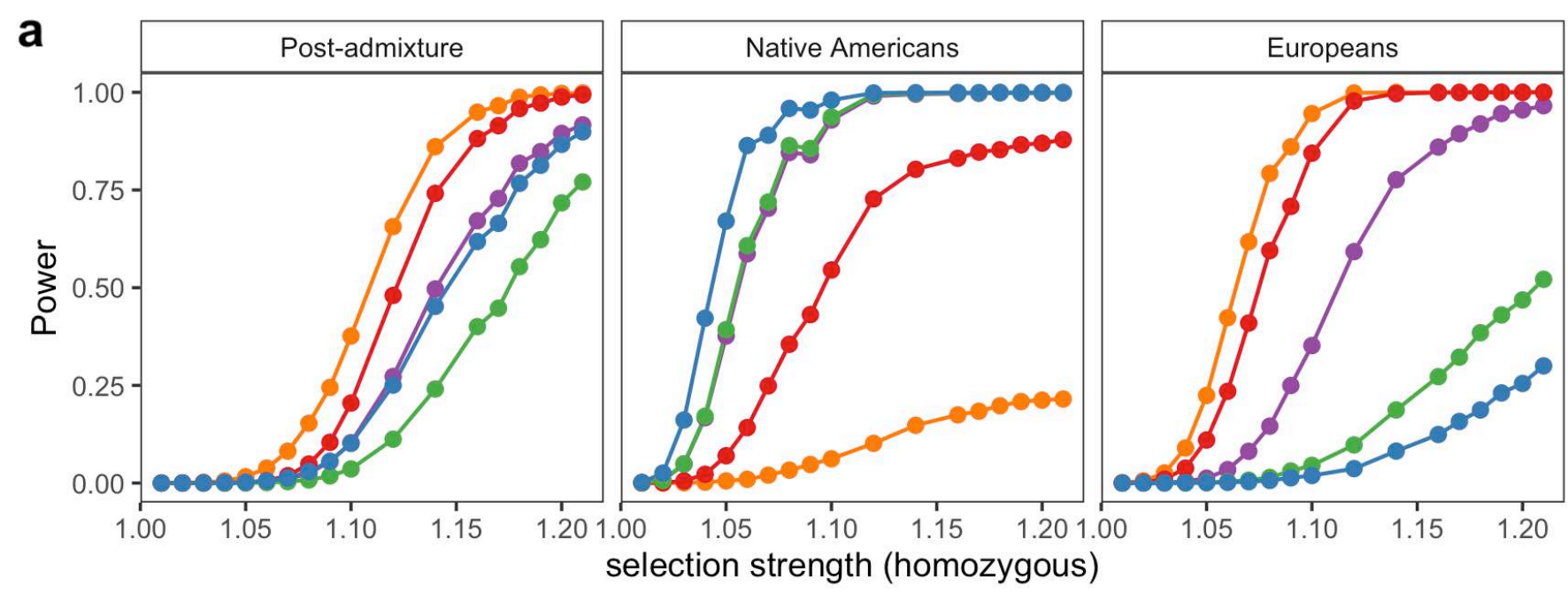

Population

$\longrightarrow$ BRA

$\rightarrow \mathrm{CHL}$

$\rightarrow \mathrm{COL}$

$\rightarrow$ MEX

$\rightarrow$ PER selection strength (homozygous)

b

\begin{tabular}{|c|c|c|c|c||}
\hline \multicolumn{5}{|c||}{ Post-admixture } \\
\hline NA & NA & NA & NA & NA \\
\hline \hline 0.01 & 0.00 & 0.00 & 0.00 & 0.99 \\
\hline \hline 0.01 & 0.01 & 0.00 & 0.00 & 0.98 \\
\hline \hline 0.02 & 0.00 & 0.00 & 0.00 & 0.97 \\
\hline \hline 0.04 & 0.01 & 0.00 & 0.00 & 0.95 \\
\hline \hline 0.07 & 0.00 & 0.00 & 0.00 & 0.92 \\
\hline \hline 0.12 & 0.01 & 0.00 & 0.00 & 0.87 \\
\hline \hline 0.16 & 0.01 & 0.00 & 0.00 & 0.83 \\
\hline \hline 0.22 & 0.01 & 0.00 & 0.00 & 0.77 \\
\hline \hline 0.28 & 0.01 & 0.00 & 0.00 & 0.71 \\
\hline \hline 0.43 & 0.00 & 0.00 & 0.00 & 0.57 \\
\hline \hline 0.54 & 0.00 & 0.00 & 0.00 & 0.46 \\
\hline \hline 0.60 & 0.00 & 0.00 & 0.00 & 0.39 \\
\hline \hline 0.63 & 0.00 & 0.00 & 0.00 & 0.37 \\
\hline \hline 0.66 & 0.00 & 0.00 & 0.00 & 0.34 \\
\hline \hline 0.68 & 0.00 & 0.00 & 0.00 & 0.31 \\
\hline \hline 0.70 & 0.00 & 0.00 & 0.00 & 0.30 \\
\hline \hline 0.72 & 0.00 & 0.00 & 0.00 & 0.27 \\
\hline
\end{tabular}

\begin{tabular}{|c|c|c|c|c|c|c|c|c|c|c|}
\hline \multicolumn{5}{|c|}{ Native Americans } & \multicolumn{5}{|c|}{ Europeans } & \\
\hline 0.01 & 0.06 & 0.00 & 0.00 & 0.93 & NA & NA & $\mathrm{NA}$ & $\mathrm{NA}$ & NA & 1.01 \\
\hline 0.01 & 0.12 & 0.00 & 0.00 & 0.87 & 0.01 & 0.00 & 0.00 & 0.00 & 0.99 & 1.02 \\
\hline 0.01 & 0.17 & 0.00 & 0.00 & 0.82 & 0.01 & 0.00 & 0.00 & 0.00 & 0.99 & 1.03 \\
\hline 0.01 & 0.24 & 0.00 & 0.00 & 0.75 & 0.01 & 0.00 & 0.01 & 0.00 & 0.98 & 1.04 \\
\hline 0.01 & 0.36 & 0.00 & 0.00 & 0.63 & 0.01 & 0.00 & 0.01 & 0.00 & 0.98 & 1.05 \\
\hline
\end{tabular}

Model chosen

\begin{tabular}{|c|c|c|c|c|c|c|c|c|c|}
\hline 0.01 & 0.45 & 0.00 & 0.00 & 0.55 & 0.01 & 0.00 & 0.02 & 0.00 & 0.97 \\
\hline 0.0 & 0.56 & 0.00 & 0.00 & 0.44 & 0.01 & 0.00 & 0.04 & 0.00 & 0.95 \\
\hline
\end{tabular}




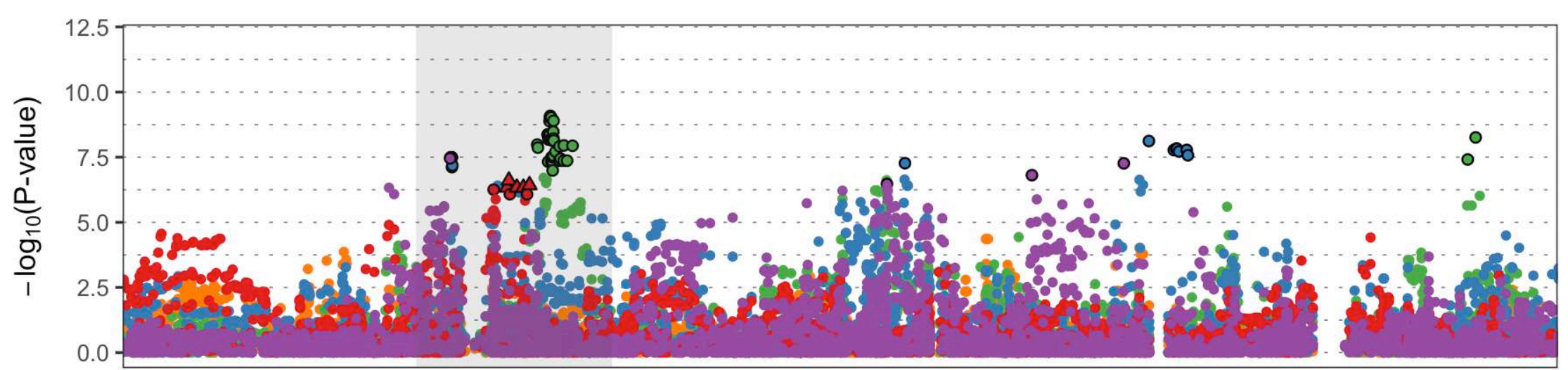

Selection model

$\triangle$ Post-admixture

○ Unclassified

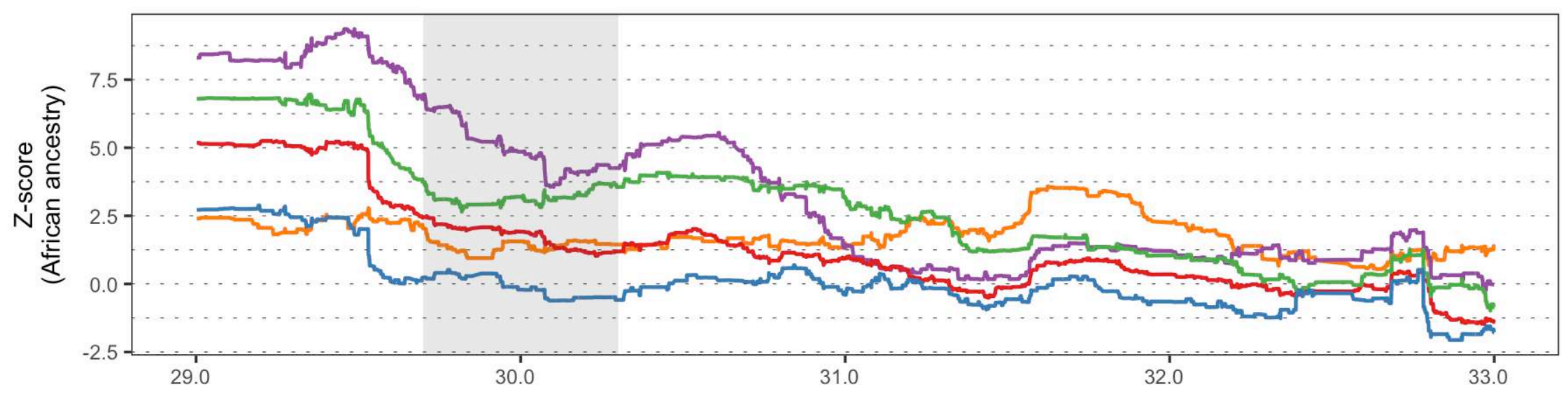

\section{Population}

- BRA

- $\mathrm{CHL}$

- COL

- MEX

- PER

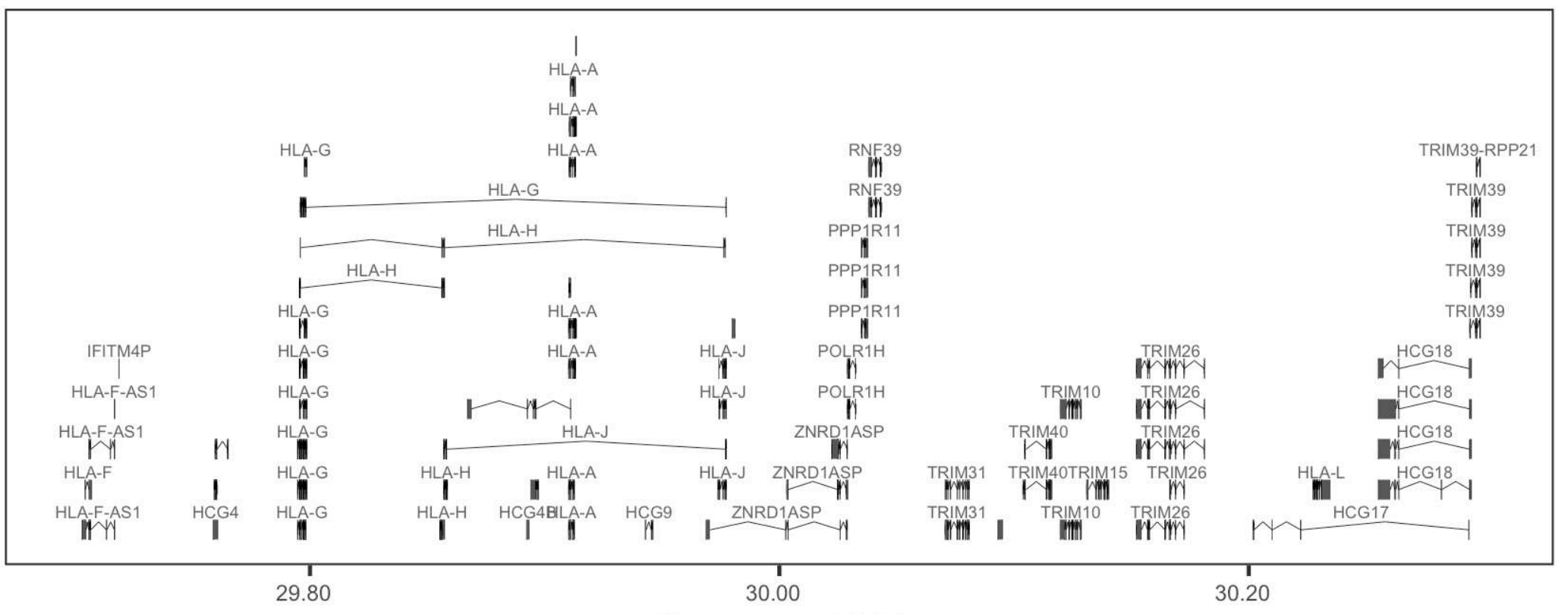

Chromosome $6(\mathrm{Mb})$ 

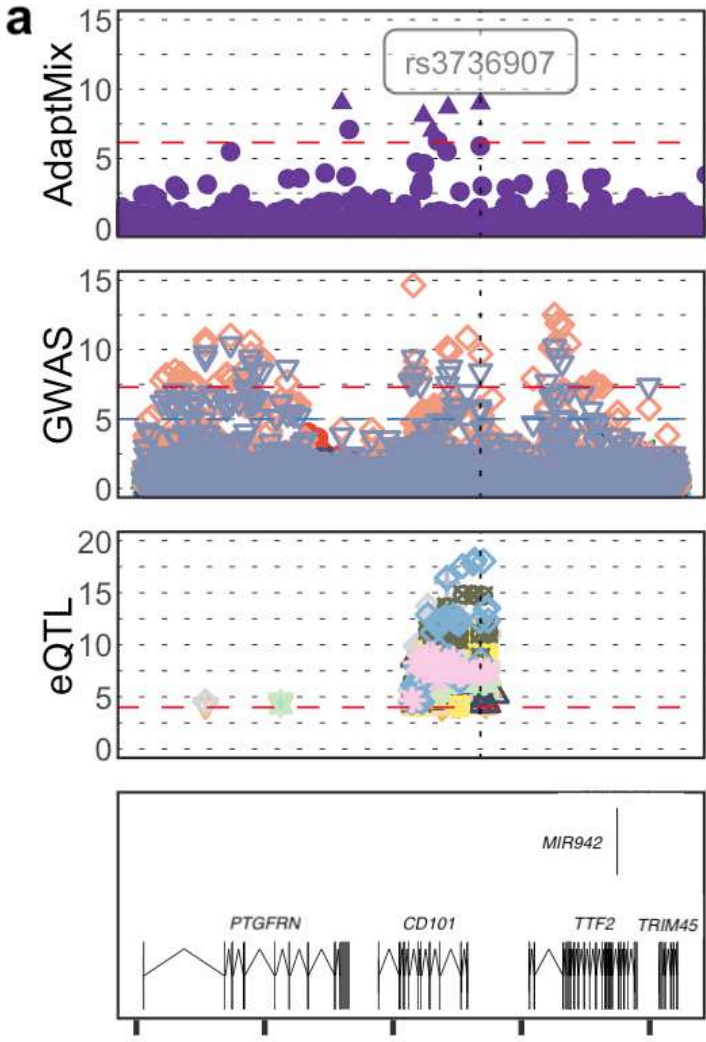

117.450117 .500117 .550117 .600117 .650 Chromosome 1 (Mb)

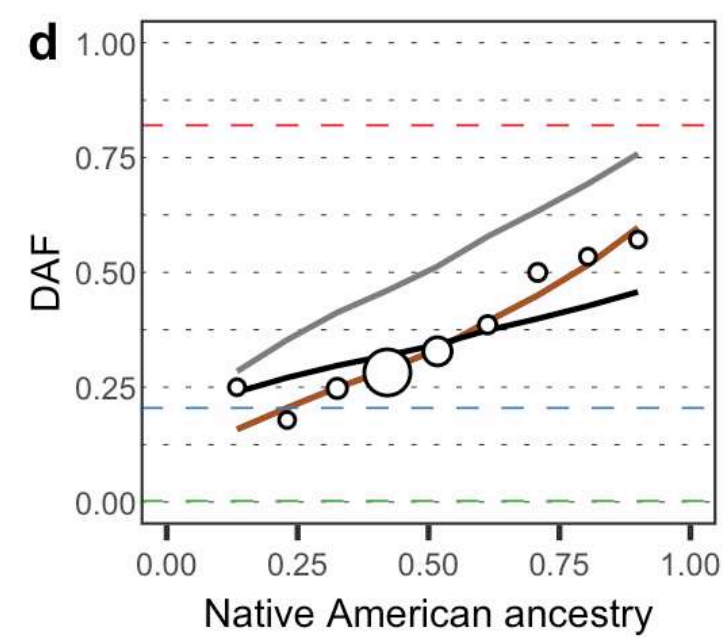

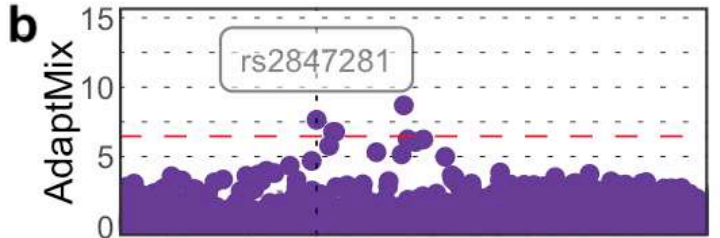
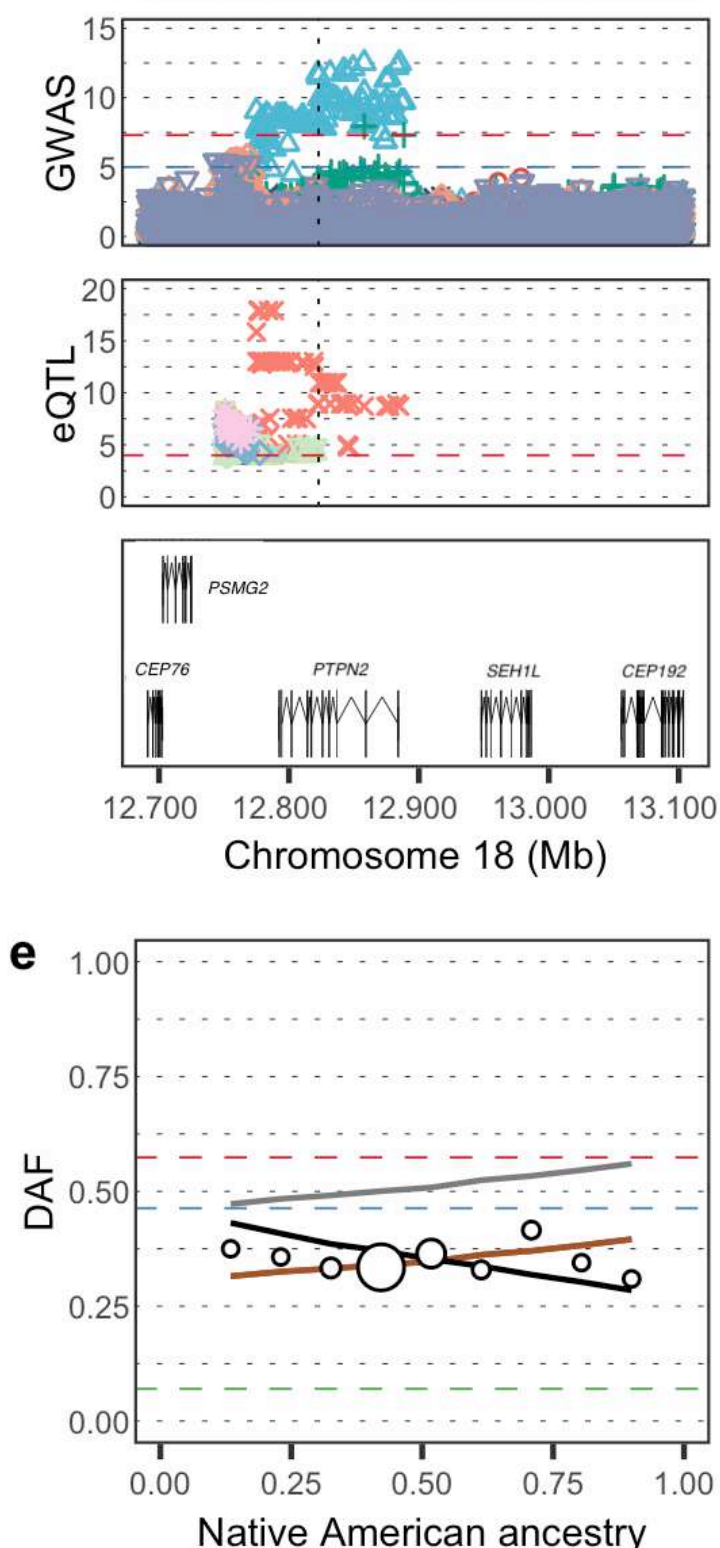

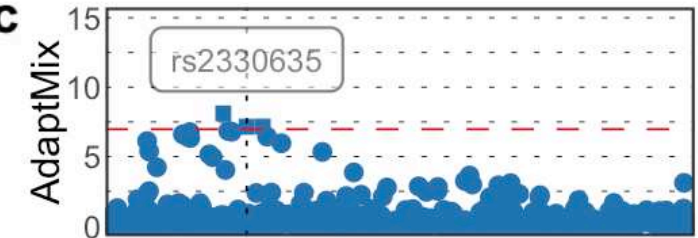

A Post-admixture

- Native Americans

- Unclassified

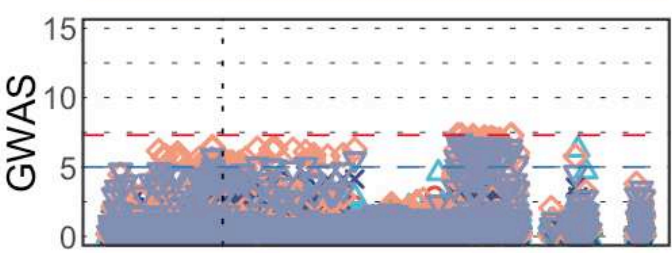

\section{- Basophil (count)}

$\Delta$ Eosinophil (count)

+ Lymphocyte (count)

Monocyte (count)

Neutrophil (count)

$\nabla$ White cell (count)

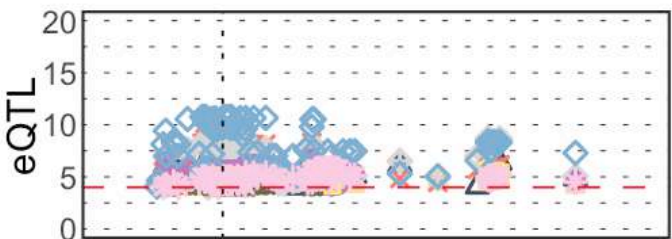

B cell, naive

$\Delta$ Monocyte, classical

Monocyte, non classical

NK cell, CD56dim CD16+

$\diamond \mathrm{T}$ cell, CD4, memory TREG
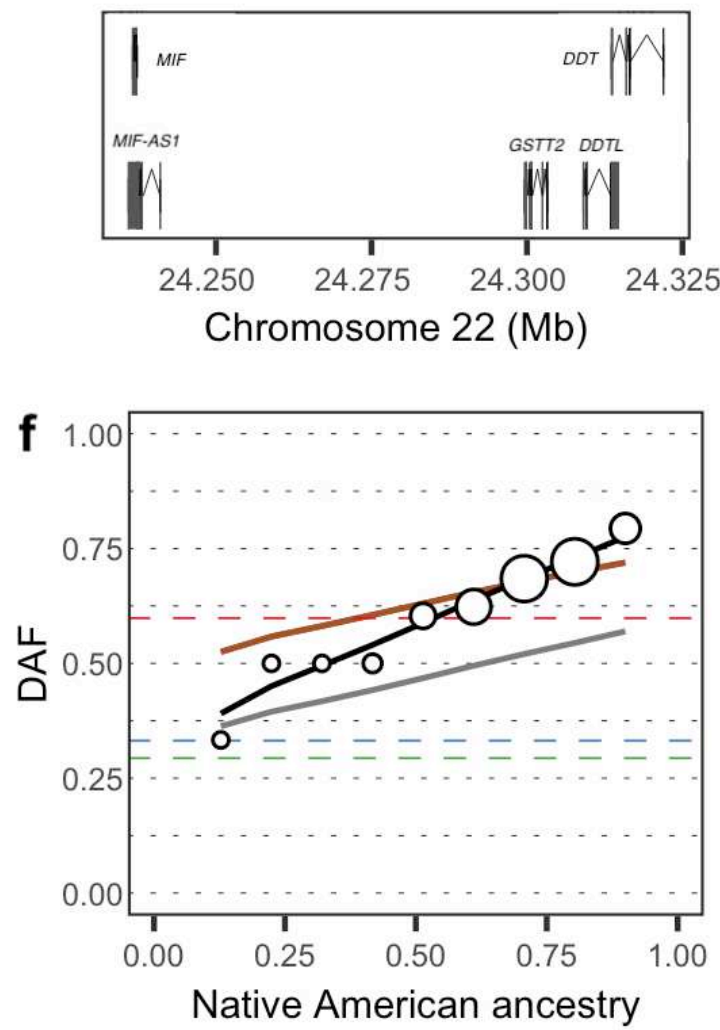

\section{Predictions}

- Post-admixture

- In parental 
a
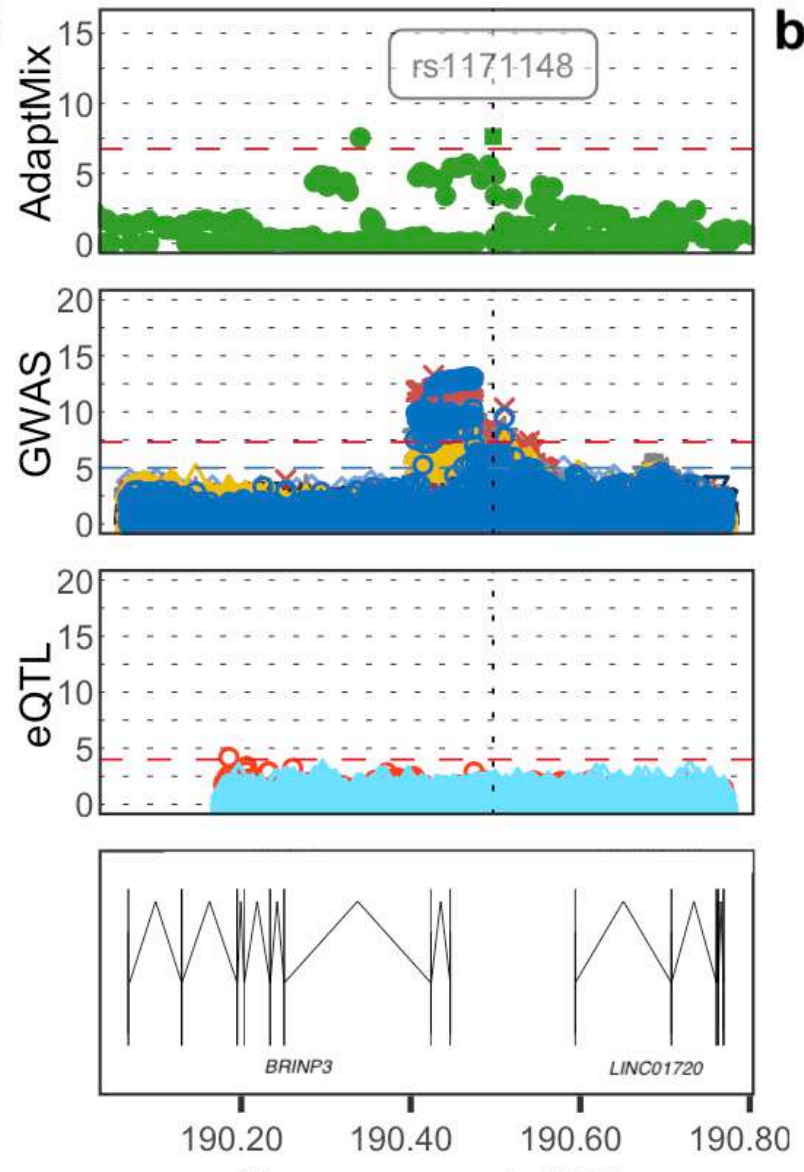

Chromosome 1 (Mb)

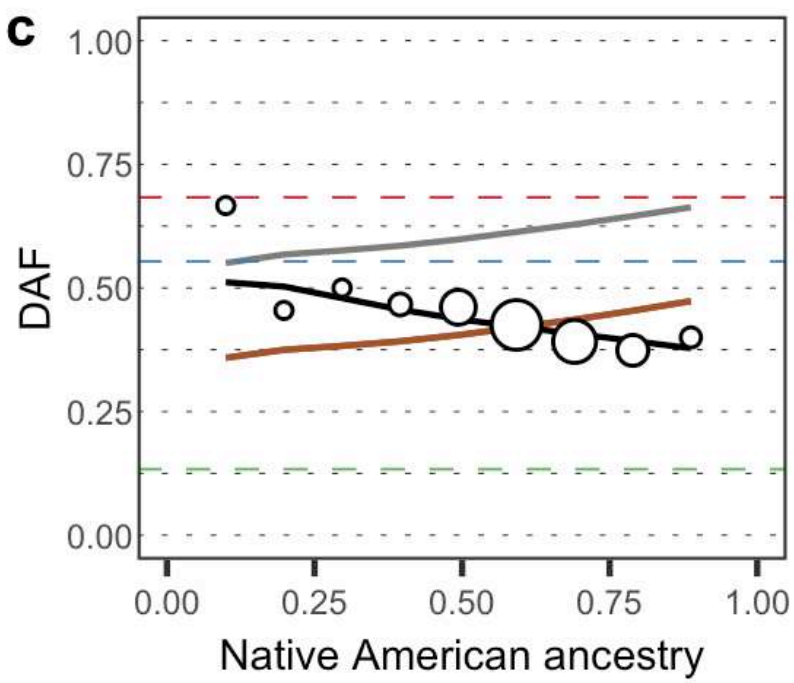

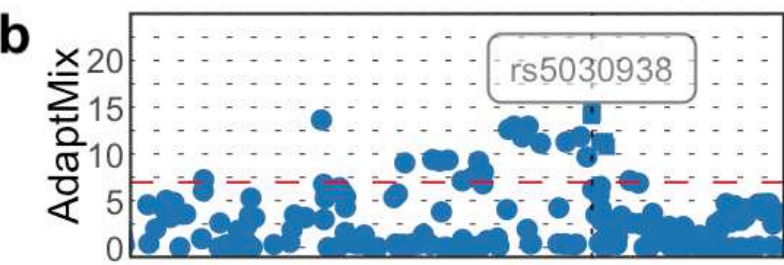
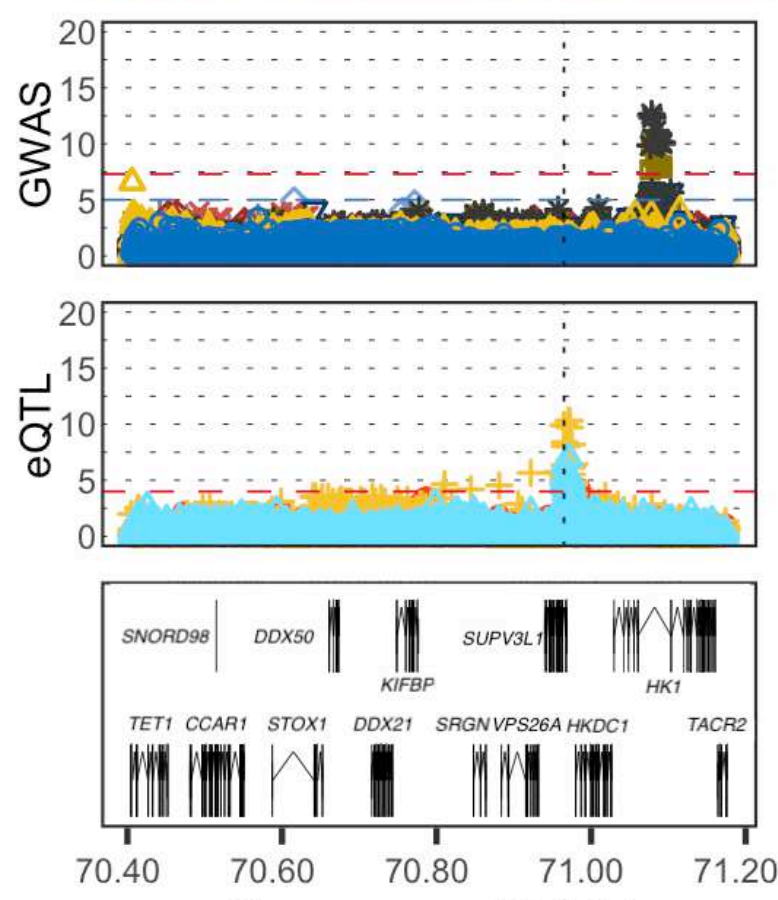

Chromosome 10 (Mb)

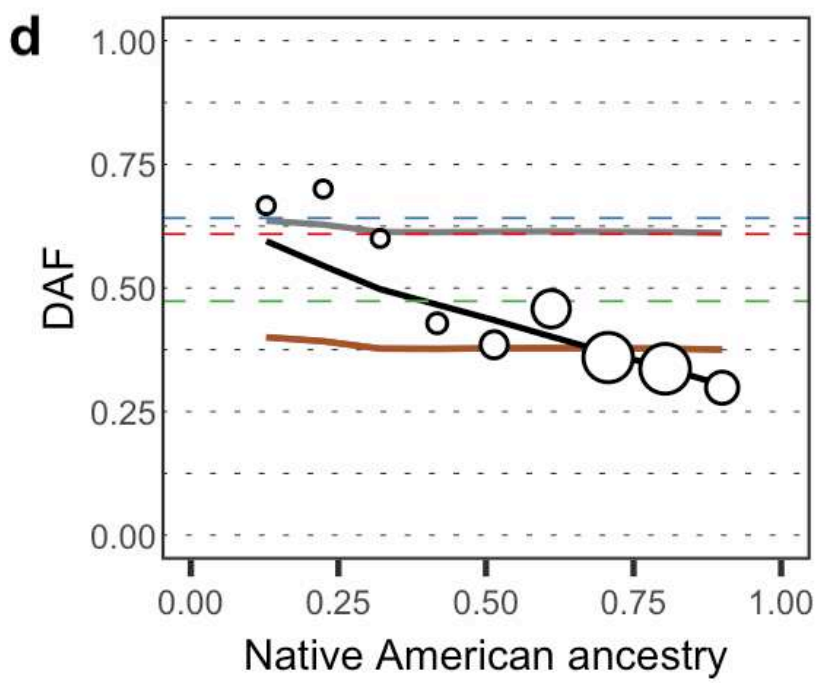

- Post-admixture

- Native Americans

- Unclassified

\section{- $\mathrm{BML} \quad \nabla \mathrm{HDL}$ \\ $\triangle$ Body fat $\otimes$ LDL \\ + WC * Cholesterol \\ $\times \mathrm{HC}$ $\stackrel{*}{\oplus} \mathrm{TG}$ \\ $\diamond \mathrm{T} 2 \mathrm{D}$ \\ - Adipose (subcutaneous) \\ Adipose (visceral omentum) \\ Liver}

\section{Predictions}

- Post-admixture

- In parental

- Neutrality 